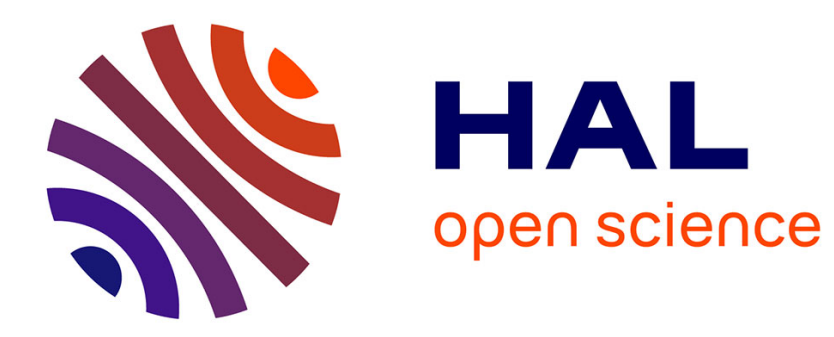

\title{
Les origines du culte des saints Cyr et Jean Jean Gascou
}

\section{- To cite this version:}

Jean Gascou. Les origines du culte des saints Cyr et Jean. 2006. halshs-00009140

\section{HAL Id: halshs-00009140 \\ https://shs.hal.science/halshs-00009140}

Preprint submitted on 17 Feb 2006

HAL is a multi-disciplinary open access archive for the deposit and dissemination of scientific research documents, whether they are published or not. The documents may come from teaching and research institutions in France or abroad, or from public or private research centers.
L'archive ouverte pluridisciplinaire HAL, est destinée au dépôt et à la diffusion de documents scientifiques de niveau recherche, publiés ou non, émanant des établissements d'enseignement et de recherche français ou étrangers, des laboratoires publics ou privés. 


\title{
Les origines du culte des saints Cyr et Jean
}

\author{
PAR JEAN GASCOU \\ Université Marc-Bloch, Strasbourg \\ INSTITUT UNIVERSITAIRE DE France \\ UMR 7044 ÉTUdE DES CIVILISATIONS DE L'ANTIQUité
}

\section{Avant-propos}

$\mathrm{D}$ ans le programme "Antiquité tardive" de l'UMR 7044 sont inscrites des recherches sur la topographie et les cultes d'Alexandrie à l'époque byzantine. Le projet le plus ample et, à ce jour le plus avancé, a trait au culte des saints martyrs Cyr et Jean, dont les reliques inhumées au martyrium de Ménouthis, sur le littoral, à 14 milles à l'est d'Alexandrie, opéraient des cures oniriques. À ces recherches participent Pauline Bringel, doctorante monitrice à l'université Marc-Bloch, Ramez Boutros, ingénieur à l'Ifao et chargé de recherche associé à l'UMR 7044, et moimême.

Le dossier hagiographique grec et latin de ces deux saints est dominé par l'œuvre du moine et sophiste Sophrone de Jérusalem ou de Damas ${ }^{1}$, composée entre 610 et

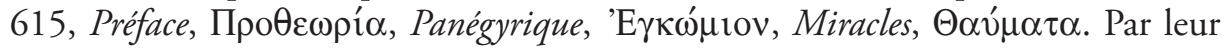
richesse historique et culturelle, les Miracles se détachent fortement de l'ensemble et attirent l'attention soutenue des spécialistes de l'Égypte byzantine. Du fait de cette sorte de célébrité, ils sont la seule pièce du corpus disponible dans une édition moderne, due à N. Fernández Marcos, Los Thaumata de Sofronio. Contribución al estudio de la Incubatio cristiana, Madrid, 1975 (ci-après FM). D'autre part, aucune de ces compositions, Miracles compris, n’a été intégralement traduite dans une langue moderne. Le dossier arabe de Cyr et Jean, d'un grand intérêt historique, est inédit. Nous croyons donc rendre service aux études en diffusant en ligne (en attendant des publications traditionnelles), à mesure qu'elles prennent forme, des rééditions ou éditions, des traductions, commentaires et études philologicohistoriques sur Cyr et Jean. C'est ici le lieu de signaler que P. Bringel vient de

\footnotetext{
${ }^{1}$ Pour la vie de Sophrone, voir Ch. von Schönborn, Sophrone de Jérusalem, vie monastique et confession dogmatique, Paris, 1972 (ci-après Schönborn); l'identité entre notre auteur et l'homonyme archevêque de Jérusalem entre 634 et 639 est discutée aux p. 239-242. Elle soulève encore quelques difficultés (voir V. Déroche, Études sur Léontios de Néapolis, Uppsala 1995, p. 25-36). Sur la date de naissance de Sophrone, vers 550 ou 560, voir S. Matter, Les poèmes anacréontiques de Sophrone de Jérusalem ou la naissance d'un genre poétique (mémoire de maîtrise, direction de B. Flusin, université de Paris IV), 1995, p. 2, n. 1 (ci-après Matter).
} 
publier une réédition électronique de la Préface et du Panégyrique comportant une traduction ${ }^{2}$.

\section{Sigles et abréviations}

\subsection{Sigles}

C: ms. Vaticanus gr. 1607

E: édition A. Mai- $P G$ 87, 3

F: ms. Berolinensis gr. 220 (Phillipps 1607)

G: ms. Glasgow UL BE.x.5 (Cunningham 64)

L: ms. Vaticanus lat. 5410, édition A. Mai-PG 87, 3

V: ms. Marcianus gr. 0595 (Mioni 583)

FM: N. Fernández Marcos, Los Thaumata de Sofronio. Contribución al estudio de la Incubatio cristiana, Madrid, 1975

\subsection{Abréviations bibliographiques}

Chuvin: P. Chuvin, Chronique des derniers païens, Paris, 1991

Delehaye, Saints d'Aboukir: H. Delehaye, "Les saints d'Aboukir ", $A B$ 30, 1911, p. 448-450

Devos: P. Devos, "Une passion grecque inédite de s. Pierre d'Alexandrie », $A B$ 83, 1965, p. 157-187

Drescher: J. Drescher, Apa Mena, a Selection of Coptic Texts relating to St. Menas, Le Caire, 1946

Duchesne: L. Duchesne, "Le sanctuaire d'Aboukir ", Bulletin de la Société archéologique d'Alexandrie 12, 1910, p. 3-14

Ehrhard: A. Ehrhard, Überlieferung und Bestand der hagiographischen und homiletischen Literatur der griechischen Kirche, I Leipzig, 1937, III Berlin-Leipzig, 1952

Flusin: B. Flusin, Saint Anastase le Perse et l'histoire de la Palestine au début du VII siècle, 2 volumes, Paris, 1992

Fournet-Gascou: J.-L. Fournet et J. Gascou, "Moines pachômiens et batellerie ", Alexandrie médiévale 2, Études alexandrines 8, 2002, p. 23-45

Gascou, Églises: J. Gascou, «Les églises d'Alexandrie, questions de méthode ", Alexandrie médiévale 1, Études alexandrines 3, Le Caire, 1998, p. 23-44

Johnson: D. W. Johnson, A Panegyric on Macarius, Bishop of Tkôw, CSCO 416, scr. copt. 42

Maraval, Lieux saints: P. Maraval, Lieux saints et pèlerinages d'Orient, Paris, 1985

Matter: S. Matter, Les poèmes anacréontiques de Sophrone de Jérusalem ou la naissance d'un genre poétique (mémoire de maîtrise, direction de B. Flusin, université de Paris IV), 1995

Montserrat: D. Montserrat, «Pilgrimage to the Shrine of SS Cyrus and John at Menouthis in Late Antiquity ", dans D. Frankfurter (éd.), Pilgrimage and Holy Space in Late Antique Egypt, Leiden, 1998, p. $257-279$

\footnotetext{
${ }^{2}$ Mes références à ce travail, que j'appelle simplement Panégyrique, suivent les divisions de l'édition
} électronique de P. Bringel (ci-après Bringel). Voir l'url: http://halshs.ccsd.cnrs.fr/halshs-00003975. 
Nissen: Th. Nissen, « De ss. Cyri et Iohannis vitae formis ", $A B$ 57, 1939, p. 65-71

Papaconstantinou: A. Papaconstantinou, Le culte des saints en Égypte, des Byzantins aux Abbassides, Paris, 2001

PLRE I: A. H. M. Jones, J. Martindale et J. Morris, The Prosopography of the Later Roman Empire, Cambridge, 1971

PLRE II: J. R. Martindale, The Prosopography of the Later Roman Empire, II, Cambridge, 1980

Sansterre: J.-M. Sansterre, «Apparitions et miracles à Ménouthis: de l'incubation païenne à l'incubation chrétienne ", Apparitions et miracles, Bruxelles, 1991, p. 69-83

Schönborn: Ch. von Schönborn, Sophrone de Jérusalem, vie monastique et confession dogmatique, Paris, 1972

Timm: S. Timm, Das christlich-koptische Ägypten in arabischer Zeit, 6 volumes, Wiesbaden, 19841992

\section{Le consensus}

Je n'ai pas trouvé d'introduction plus concise et complète à l'histoire du culte de Cyr et Jean qu'un passage du bollandiste Hippolyte Delehaye ${ }^{3}$. Ces pages cristallisent le consensus qui prévalait alors et continue à l'emporter ${ }^{4}$.

"Moins anciennes que celles du grand pèlerinage de la Maréotide ${ }^{5}$ sont les origines du sanctuaire des saints Cyr et Jean à Menouthis, à deux milles de Canope. Le temple d'Isis, qui s'élevait dans cette localité, avait longtemps été fort fréquenté. Il fut fermé, et une église, dédiée aux Évangélistes, construite dans la localité. Mais cela ne suffit pas à faire oublier l'ancien culte et à étouffer les germes de paganisme qui subsistaient encore. Cyrille d'Alexandrie crut en avoir raison en installant, près de l'ancien temple d'Isis, le culte des martyrs. Il fit ouvrir, dans la basilique de Saint-Marc d'Alexandrie, le tombeau des saints Cyr et Jean, avec l'intention de transporter un des saints corps à Menouthis. Dans l'impossibilité de distinguer à qui des deux saints appartenaient les squelettes, il les fit enlever tous les deux. Il y eut une translation solennelle, à l'occasion de laquelle Cyrille prit plusieurs fois la parole. Nous possédons encore le texte de ces allocutions. Sur les deux martyrs, on savait peu de chose. Les récits qui nous sont parvenus sont bien vagues et faits, on le sent, sur des données maigres et incertaines. On dit que Cyr était moine, et Jean soldat. Le premier exerçait d'abord la médecine, et l'on

${ }^{3}$ Les origines du culte des martyrs, Subsidia hagiographica 20, Bruxelles, 1933, p. 223-224.

${ }^{4}$ Je n'ai pas pu me procurer C. Cozzolino, Origine del culto dei santi Ciro e Giovanni in Oriente e in Occidente, Jérusalem, 1976. Pour une illustration de la vue traditionnelle, voir l'article influent de R. Herzog, "Der Kampf um den Kult von Menuthis ", Pisciculi Franz Joseph Dölger dargeboten, Münster, 1939, p. 117-124, et, récemment, D. Montserrat, «Pilgrimage to the Shrine of SS Cyrus and John at Menouthis in Late Antiquity ", dans D. Frankfurter (éd.), Pilgrimage and Holy Space in Late Antique Egypt, Leiden, 1998, p. 257-279 (ci-après Montserrat).

${ }^{5}$ Le P. Delehaye fait allusion à Saint-Mènas, sanctuaire dont les origines, d'après les investigations archéologiques contemporaines, ne sont pas aussi anciennes qu'on ne le croyait alors. 
montrait à Alexandrie, englobé dans l'église des Trois-Enfants, le cabinet où il avait donné ses consultations - une légende qui se forma, sans doute lorsque les miracles de Menouthis eurent établi sa réputation de guérisseur. Ce que nous savons de l'histoire de Menouthis, au cours du v viècle, nous oblige à conclure que la présence des deux martyrs n'opéra pas instantanément les effets que Cyrille en attendait, et qu'il fallut d'autres interventions pour extirper les restes de paganisme et donner au nouveau culte tout son essor. Ce fut sans doute durant le $\mathrm{vI}^{\mathrm{e}}$ siècle que la vogue du sanctuaire s'accrut dans les grandes proportions. Dans les premières années du siècle suivant, Sophrone de Jérusalem, en reconnaissance de sa propre guérison, fit un recueil des miracles obtenus par l'intercession des SS. Cyr et Jean, compilation considérable et infiniment curieuse, qui fait défiler devant nous la foule pressée des pèlerins venus à Menouthis, pour obtenir la guérison de leurs maux. On sait que le nom d'Aboukir, qui n'est autre qu'une déformation arabe de celui de S. Cyr, 'A $\beta \beta \alpha \kappa \hat{\rho} \rho \varsigma_{s}$, a remplacé le nom de Menouthis et demeure, aujourd'hui encore, le témoin de la transformation accomplie en ces lieux par le culte des martyrs. On ne peut déterminer exactement l'époque de la ruine du sanctuaire. Les reliques des saints furent transportées à Rome et déposées dans une petite église, située sur la rive droite du Tibre, à la hauteur de la basilique de Saint-Paul. On l'appelle encore Santa Passera, nouvel et singulier dérivé de 'A $\beta \beta \alpha \kappa v ̂ \rho о \varsigma$ ".

Ailleurs dans l'ouvrage ${ }^{6}$, le P. Delehaye mentionne trois jeunes filles associées, avec leur mère Athanasie, au martyre et à certains aspects du culte de Cyr et Jean, Théodote, Théoctiste et Eudoxie. Cyr et Jean les auraient assistées au moment de leur épreuve. Après les exécutions, les quatre femmes et les deux hommes auraient été inhumés au martyrium de saint Marc, dans des tombeaux distincts. Un puits miraculeux conservait à Saint-Marc le souvenir des héroïnes.

Dans sa reconstitution de la personnalité des deux martyrs, de la mise en place et du développement de leur culte funéraire, le P. Delehaye a mêlé dans un récit homogène, mais assorti de nuances et aveux d'incertitude qui inspirent confiance, les données du corpus sophronien, de deux ou trois allocutions (Oratiunculae) attribuées à s. Cyrille, de deux biographies ou Vies grecques anciennes. Or, si ces sources concordent pour vanter et promouvoir le culte reçu au début du vir ${ }^{\mathrm{e}}$ siècle à Ménouthis, elles conservent, sous des formes résiduelles trahissant des censures ou réécritures, les revendications d'autres sanctuaires alexandrins, égyptiens ou étrangers, et donc les traces d'une dissémination précoce du culte peu agréable au clergé de Ménouthis. D'autre part, le P. Delehaye n'a pas mis à concours un texte dont il est l'éditeur justement célèbre, le Synaxaire de la Grande Église de Constantinople, qui oblige à poser en d'autres termes la question des origines. Une histoire du culte de Cyr et Jean devrait donc se fonder sur une discussion littéraire et une mise en perspective de l'ensemble du corpus hagiographique: c'est ce projet 
que nous avons en tête dans les moyen et long termes. Voici cependant une vue abrégée de nos résultats.

\section{Le dossier hagiographique}

\subsection{Le ms. Vaticanus gr. 1607 (C)}

La partie grecque du dossier se trouve en entier dans un manuscrit d'origine italo-grecque de la fin du $\mathrm{x}^{\mathrm{e}}$ s., le Vaticanus gr. 1607. Comme il provient de l'abbaye basilienne de Grotta Ferrata (Crypta Ferrata), nous l'appelons C. C'est un corpus liturgique presque entièrement consacré à nos saints ${ }^{7}$ où l'on trouve successivement :

- Une vie acéphale de Cyr et Jean ou V1 (fo $\left.1^{\mathrm{r}}-4^{\mathrm{r}}\right)$

- Trois Oratiunculae placées sous le nom de s. Cyrille $\left(\mathrm{f}^{\mathrm{o}} 4^{\mathrm{v}}-5^{\mathrm{v}}\right)$

- Une deuxième vie de Cyr et Jean ou V2 (fo $\left.6^{\mathrm{r}-}-12^{\mathrm{v}}\right)$

- Le corpus sophronien, se subdivisant en

Préface et Panégyrique (fo $\left.13^{\mathrm{r}}-33^{\mathrm{v}}\right)$,

Miracles de Cyr et Jean ( $\mathrm{f}^{\mathrm{o}} 33^{\mathrm{v}}-150^{\mathrm{v}}$ )

$\mathrm{Au}$ vu des matériaux qu'il incorpore, on peut rapprocher $\mathrm{C}$, entre autres, du ms. copte Pierpont Morgan 590 ( $\mathrm{IX}^{\mathrm{e}}$ s.), consacré à saint Mènas, l'autre grand saint égyptien de l'époque, où se succèdent une Passion, des Miracles et un Panégyrique ${ }^{8}$. Ces recueils réunis autour d'un saint étaient destinés à la lecture publique aux jours de fête? .

Tout ce qui dans C a trait à Cyr et Jean a été édité en 1840 par le cardinal Angelo $\mathrm{Mai}^{10}$. À l'exception des Oratiunculae, incorporées aux œuvres de Cyrille, cette publication est passée dans le volume 87,3 de la Patrologie Grecque $(P G)^{11}$. Au cours de ce transfert, un savant anonyme est intervenu à plusieurs reprises, et le

${ }^{7}$ Les quatre derniers feuillets ( $f^{\circ}$ 151-154) sont cependant meublés par diverses petites pièces sans rapport avec le gros du corpus, dont un traité sur le jeûne par Dôrothée de Gaza, auteur ascétique dont le floruit se place au milieu du vi ${ }^{\mathrm{e}}$ s., copié à la même époque que le reste.

${ }^{8}$ J. Drescher, Apa Mena, a Selection of Coptic Texts relating to St. Menas, Le Caire, 1946 (ci-après Drescher).

${ }^{9}$ C'est ainsi qu'A. Ehrhard, Überlieferung und Bestand der hagiographischen und homiletischen Literatur der griechischen Kirche (...), III, p. 899-900 (voir p. 895), classe C (ci-après Ehrhard). Sur l'usage de ce genre de composition dans la prédication et la lecture, voir P. Maraval, Lieux saints et pèlerinages d'Orient, Paris, 1985, p. 217-218, l'auteur citant même des cas de joutes oratoires en l'honneur de sainte Thècle, analogues aux compétitions épidictiques profanes (ci-après Maraval, Lieux saints).

${ }^{10}$ Spicilegium romanum 3, 1840, p. 1-696 (corpus sophronien) : 4, 1840, p. 230-241 (deuxième Vie), 242-248 (première Vie), 248-252 (Oratiunculae).

${ }^{11}$ Col. 3424-3676. Pour les Oratiunculae, voir ci-dessous 3.3. 
plus souvent à bon escient, dans le texte de $\mathrm{Mai}^{12}$. Comme FM, nous désignons le résultat sous le sigle $\mathrm{E}$. C abonde en graphies phonétiques et formes corrompues, en lacunes, interversions de phrases ou de passages, corrections et interpolations. Ces déficiences sont en partie compensées par des ménologes grecs qui ont transmis, d'une manière sélective ou anthologique, mais parfois plus satisfaisante du point de vue textuel, certaines pièces de C. La plupart de ces manuscrits étaient inconnus de Mai et restent inédits, mais nous en avons tenu compte, de même que nous avons utilisé un corpus latin d'origine romaine conservé par un apographe daté de 1600, le ms. Vaticanus lat. 5410 (ci-après L) ${ }^{13}$. Traduit du grec par l'archidiacre et consiliarius Boniface (actif entre 654 et 704) ${ }^{14}$ et le célèbre Anastase le Bibliothécaire (actif dans la $2^{\mathrm{e}}$ moitié du Ix $\mathrm{x}^{\mathrm{e}}$., avant 886 ), il témoigne de la diffusion précoce à Rome, à compter du VII ${ }^{\mathrm{e}}$ siècle, du culte de Cyr et Jean ${ }^{15}$. Son édition, due aussi à Mai, figure en regard de $\mathrm{E}$ dans $P G 87,3^{16}$. Aujourd'hui acéphale, $\mathrm{L}$ contenait à l'origine les mêmes pièces que $\mathrm{C}$, et à peu près dans le même ordre. Ses leçons impliquent un original grec divergeant parfois de $\mathrm{C}$, mais sur des points mineurs. Malgré sa mutilation initiale, $\mathrm{L}$ a une grande autorité ${ }^{17}$ et permet souvent de combler les lacunes de $\mathrm{C}$ et d'en corriger les parties corrompues.

\subsection{Les Vies}

Séparées par les Oratiunculae, se présentent au début de C deux courtes Vies que j'appelle V1 et V $2^{18}$. On les a parfois attribuées à Sophrone, mais cette opinion

${ }^{12}$ Ceci a été remarqué par J. Duffy, « Observations on Sophronius Miracles of Cyrus and John », JThS 35, 1984, p. 77, pensant à un collaborateur de l'abbé J.-P. Migne; voir aussi Th. Nissen, « De ss. Cyri et Iohannis vitae formis ", $A B$ 57, 1939, p. 65, n. 1 (ci-après Nissen).

${ }^{13}$ Le manuscrit d'où fut tirée la copie, aujourdhui disparu, datait de 1204 et était conservé dans l'église romaine de Sainte-Marie in via Lata.

${ }^{14}$ Lactivité de Boniface se limite à la traduction des douze premiers miracles sophroniens. Voir sur L, A. Poncelet, Catalogus codicum hagiographicorum latinorum Bibliothecae Vaticanae, Subsidia hagiographica 11 Bruxelles, 1910, p. 130-131; sur Boniface, W. Berschin, "Bonifatius Consiliarius, ein römischer Übersetzer in der byzantinischen Epoche des Papsttums ", Lateinische Kultur im VIII. Jahrhundert. Traube-Gedenkschrift, St. Ottilien, 1989, p. 25-40.

${ }^{15}$ De fait, trois églises dédiées à nos saints sont attestées à Rome dès le Haut Moyen Âge (D. Nuzzo, "Egiziani a Roma in età tardoantica, osservazioni sulle fonte epigrafiche e letterarie ", Vetera Christianorum 36, 1999, p. 228). D'après L, Rome prétendait avoir reçu les reliques lorsque Alexandrie était sous domination arabe ( $P G$ 87, 3, col.3675-3676). Je n'ai pas l'intention de discuter cette question, mais un miracle du dossier arabe de Cyr et Jean va dans le sens de la cause romaine (il fait état d'une icône et non plus des reliques).

${ }^{16}$ Le corpus sophronien latin est édité par Mai sous le texte grec dans Spicilegium romanum 3, 1840, p. 1-696. On trouve la deuxième Vie latine dans Spicilegium romanum 4, 1840, p. 253-263. La traduction des Oratiunculae par Anastase, Spicilegium romanum 4, 1840, p. 263-266, est toutefois reprise dans les œuvres de Cyrille, au volume 77 de $P G$ (voir ci-dessous 3.3).

${ }^{17}$ Sur la position de L dans le stemma codicologique, voir Bringel, introduction au Panégyrique.

${ }^{18}$ Elles se succèdent dans $\mathrm{E}$ dans l'ordre inverse de C. 
n'a plus cours, et la cause me paraît entendue ${ }^{19}$. Selon les règles du genre, elles comportent un préambule oratoire, une biographie des héros, des développements sur leur procès, leur martyre en compagnie des saintes filles, et la translation de leurs reliques en leur martyrium de Ménouthis à l'initiative de Cyrille. Sur le plan historique, il y a entre elles un accord de façade, mais, dans un état originel dont nous essaierons de nous faire une idée, chacune défendait sur Cyr et Jean des points de vue inconciliables.

\subsubsection{La première Vie}

V1 (BHG I 470; CPG III 7674) est acéphale dans $\mathrm{C}$ et a disparu dans la mutilation initiale de $\mathrm{L}$, mais est restituée au complet par trois manuscrits, le Kutlumusiu 37, fo $263^{\mathrm{v}}-266^{\mathrm{v}}$, le Lavra 327, fo $84^{\mathrm{r}}-88^{\mathrm{r}}$, et l'Ambrosianus gr. F 144 sup., f $144^{\mathrm{r}}-146^{\mathrm{r} 20}$. J'en prépare une réédition d'après le Kutlumusiu 37 . En raison d'emprunts que j'y ai constatés à la version longue des Acta de l'évêque alexandrin Pierre $\mathrm{I}^{\mathrm{er}}(\dagger 25$ novembre 311$)$, version qui ne peut guère être antérieure au $\mathrm{VI}^{\mathrm{e}}$ $s .{ }^{21}$, il y a lieu de penser que V1, dans son état actuel, n’a pas été rédigée avant le $\mathrm{VI}^{\mathrm{e}}$ s. assez avancé ou même le début du $\mathrm{VII}^{\mathrm{e}}$. Une datation plus précise pourrait se tirer du ms. Kutlumusiu 37 qui, à la suite de V1, donne aux fo 266 v $-269^{\mathrm{v}}$ une collection de cinq miracles de Cyr et Jean, ne devant rien à Sophrone (BHG I 479i). Or l'intitulé de cet ensemble organiquement lié à V1 comporte une datation par l'an 24 d'Héraclius (octobre 634/octobre 635):

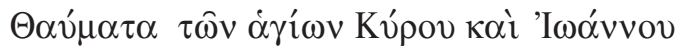

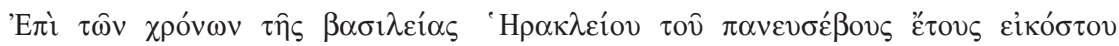

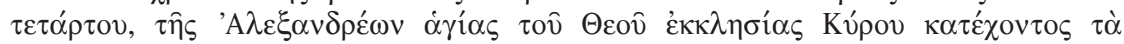

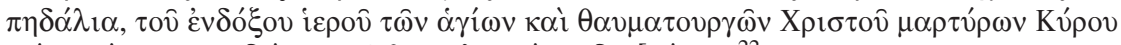

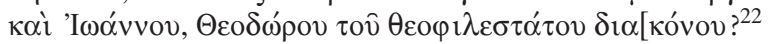

${ }^{19}$ Voir $C P G$ III n ${ }^{\text {os }} 7673$ et 7674 , et Nissen, p. 65-71, sp. p. 65-66.

${ }^{20} P G$ 87, 3, col. 3689-3696. Voir Ehrhard I, p. 534 et III, p. 899-900 (description conforme à l'ordre réel de C). Le ms. Kutlumusiu 37, f $263^{\vee}-266^{v}$, définit cette composition comme $\dot{\gamma} \gamma \kappa \omega ́ \mu ı v$, les deux autres ont $\mu \alpha \rho \tau$ vípiov.

${ }^{21}$ Voir W. Telfer, "Peter of Alexandria and Arius", $A B$ 67, 1949, p. 117-130, spéc. p. 118-122. Pour la version longue des Acta Petri, je me réfere à P. Devos, "Une passion grecque inédite de s. Pierre d'Alexandrie ", $A B$ 83, 1965, p. 157-187 (ci-après Devos). Leur influence se détecte dans une comparaison entre l'évêque martyr et l'apôtre homonyme (Devos, p. 172; comparer $P G$ 87, 3, col. 3692), dans les termes flétrissant Arius et la prétendue déposition de l'hérésiarque, attribuée au même évêque (Devos, p. 164; comparer $P G$ 87, 3, col. 3692). Le récit des funérailles de Cyr et Jean et de leurs compagnes est tributaire des Acta Petri sur plusieurs points, comme l'implication du mausolée de Saint-Marc (Devos, p. 171, 175; comparer $P G$ 87, 3, col. 3693), le rôle du peuple alexandrin et

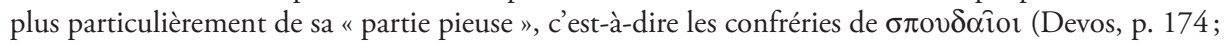
comparer $P G 87,3$, col. 3693).

${ }^{22}$ Le texte est interrompu par une lacune de quatre lignes. On voit que le célèbre évêque Cyrus (631/642) est mentionné sans faveur marquée, sans doute en raison de son rôle dans la promotion du monothélisme, qui lui aliéna les chalcédoniens sans satisfaire les Coptes. Théodôros devait être alors diacre et directeur (économe?) du martyrium, un des successeurs de Christodôros, héros du miracle sophronien $\mathrm{n}^{\circ} 8$. 
Si le narrateur de V1 tait ce qu'il doit aux Acta Petri, il avoue des sources. Indiquant, en effet, comme " cause " de la translation à Ménouthis, les "illusions ", $\varphi \alpha v \tau \alpha \sigma i \alpha$, et autres duperies d'un démon féminin, il se réfère aux ouvrages de

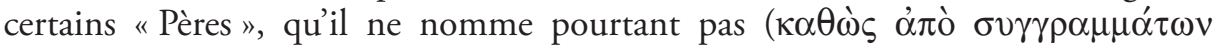

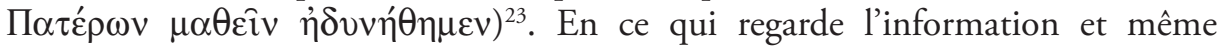
l'expression, V1 concorde souvent avec le Panégyrique ${ }^{24}$, mais s'en écarte parfois, si bien que l'on ne peut se prononcer en toute certitude sur les rapports entre les deux compositions. Je reviendrai sur ce problème à propos du Panégyrique.

Quelle qu'en soit la solution, V1 enregistre ce que le clergé de Ménouthis souhaitait que l'on crût sur Cyr et Jean à la fin du $\mathrm{VI}^{\mathrm{e}}$ ou au début du $\mathrm{VII}^{\mathrm{e}}$ s., en somme une orthodoxie cultuelle. Que nous dit-elle d'historique? Cyr était moine, Jean, soldat. Ils vivaient tous deux à Canope (d'où venaient aussi les vierges). Ils furent arrêtés sous Dioclétien, au temps où Syrianos gouvernait Alexandrie et Kasios $^{25}$ était grand-prêtre de Canope. Après leur procès au tribunal de Syrianos, ils furent martyrisés le 6 mecheir (31 janvier) 312, en compagnie de Théodote, Théoctiste ${ }^{26}$, Eudoxie, et leur mère Athanasie. Les corps furent inhumés au tombeau de saint Marc. Bien plus tard, l'évêque Cyrille (412/444) apprit qu'un démon oraculaire nommé Ménouthè sévissait à Ménouthis. Il voulut l'éradiquer. Un ange lui dit en songe que les reliques de Cyr et Jean, dont il lui révéla l'emplacement à Saint-Marc, auraient ce pouvoir. Cyrille les transporta à l'église des Évangélistes de Ménouthis en un cercueil unique. Aussitôt les prêtres du démon demandèrent le baptême, l'idole et son temple disparurent sous le sable et la mer, et les malades du monde entier se mirent à affluer au mausolée de Cyr et Jean.

Rapportée aux débats christologiques de l'époque, V1 est neutre. Ainsi s'explique, au témoignage du Synaxaire copte ${ }^{27}$ et d'un exemplaire traduit (non sans adaptations) en arabe en cours d'étude par R. Boutros, qu'elle ait eu cours dans l'Égypte médiévale.

${ }^{23} P G$ 87, 3, col. 3693.

${ }^{24}$ Voir Nissen, p. 66: etsi argumentum eius ad Sophronii laudationem proxime accedit.

${ }^{25}$ Ces noms ont disparu dans la lacune de C, mais sont dans les autres ms. (le Lavra 327 a toutefois Kasinos au lieu de Kasios, ce qui se rapproche de la forme Kasianos de l'exemplaire de V2 transmis par C).

${ }^{26}$ L'incipit du ms. Kutlumusiu 37, le Synaxaire copte au 6 mecheir (PO 11, p. 796-797) et la version arabe de V1 donnent à Théoctiste le nom de Théopiste. Deux saintes de ce nom étaient vénérées en Égypte, mais je ne vois pas dans quelles conditions le nom de Théopiste a pu s'introduire dans les traditions de Ménouthis (voir G. Nachtergael et A. Papaconstantinou, « Une lampe d'Égypte au nom de sainte Théopistè ", Ricerche di Egittologia e di Antichità Copte, 6, 2004, p. 87-89).

${ }^{27}$ PO 17, p. 621-622 (la destruction du sanctuaire est décrite dans les mêmes termes que V1, même si le nom de Ménouthis a été censuré). 


\subsubsection{La deuxième Vie}

V2 (BHG I 469; CPG III 7673) a perdu dans C son second feuillet ${ }^{28}$, mais est préservée dans son intégralité par un manuscrit de 1022, le Mosquensis 162 (Vladimir 380), f $104^{\mathrm{r}}-110^{\mathrm{r}}$, d'origine athonite (ci-après $\mathrm{M}$ ), et un ménologe attribué $\mathrm{au} \mathrm{x}^{\mathrm{e}}$ ou au XI ${ }^{\mathrm{e}}$ s., le Glasgow UL BE.x.5 (Cunningham 64), f $320^{\mathrm{r}}-325^{\mathrm{v}}$ (ci-après $\mathrm{G})^{29}$. Un témoin ancien de V2 est offert par L, f $\mathrm{f}^{\circ}$ 32-38 (BHL I 2077), dont les divergences par rapport à $\mathrm{C}$ sont minimes ${ }^{30}$. On en a aussi une recension métaphrastique (ci-après Met.) ${ }^{31}$. Une recension postmétaphrastique est procurée par un ménologe du XII ${ }^{\mathrm{e}}$ s., le Berolinensis gr. 220, f 202-214 (Phillipps 1623 ; ciaprès $\mathrm{F})^{32}$. Th. Nissen en a publié le début, qui restitue ce qui manquait dans $\mathrm{C}$, et c'est lui qui a montré ce que ce texte devait à Met. Le même Nissen a enfin établi que le Bollandiste O. Zylius, pour sa vie latine de Cyr et Jean, aux Acta sanctorum du 31 janvier, p. 1084-1088, avait utilisé F ou son « jumeau ${ }^{33}$.

Je ne sais quelle recension de V2 enregistre l'Ambrosianus gr. D 107 sup., fo $320^{\mathrm{v}}-327^{\mathrm{v}}$ (Corfou; fin du XIV s.) ${ }^{34}$.

C'est un écrit décousu, squelettique sur des points essentiels (translation, débuts du culte), mais encombré de digressions longues et étranges. Si l'on peut rendre compte de l'histoire du martyrium alexandrin des Trois-Enfants (Hébreux), qui occupe les premiers feuillets ${ }^{35}$, le long développement sur l'ascète scétiote Sénouphios (aition d'une fête alexandrine de l' "Image ") ${ }^{36}$ n'a rien à voir avec Cyr et Jean. La description de Canope, ses monuments païens et ses embellissements, dont la construction d'une église des Saints-Apôtres, par Théophile, oncle et prédécesseur

\footnotetext{
${ }^{28} P G$ 87, 3, col. 3677-3689.

${ }^{29}$ Voir sur ce codex B. Flusin, Saint Anastase le Perse, I, Paris, 1992 (ci-après Flusin), p. 19. Il s'agit d'un ménologe de janvier, en désordre et lacunaire selon $\mathrm{D}$. Weston, conservateur des fonds spéciaux à la University Library de Glasgow. Les dates proposées sont le $\mathrm{x}^{\mathrm{e}} \mathrm{s}$. (F. Halkin, « Un nouveau ménologe de janvier dans un manuscrit de Glasgow ", $A B 75,1957$, p. 66-71), et le XI ${ }^{\text {e }}$ dans le catalogue de S. I. C. Cunningham, Greek Manuscripts in Scotland, Edinburgh, 1982, p. 16, n 64.

${ }^{30}$ Pour la restitution d'une lacune initiale de V2 dans L, voir $A B$ 8, 1889, p. 95-96.

${ }^{31} P G$ 114, col. 1233-1249.

32 Sur la date de ce codex, $\mathrm{XII}^{\mathrm{e}}$ s., voir F. Halkin, $A B$ 93, 1975, p. 422, et Flusin, p. 18. C'est le deuxième tome d'un ménologe de janvier.

${ }^{33}$ Nissen, p. 68-71 (gemellus, p. 67). Le texte, d'après les constatations de Nissen, suit C jusqu'au $\mathrm{f}^{\circ}$ 204 puis, jusqu’à la fin, concorde avec Met. (Nissen, p. 67).

${ }^{34}$ Voir C. Pasini, Inventario agiografico dei manoscritti greci dell'Ambrosiana, Subsidia hagiographica 84, Bruxelles, 2003, p. 64-66. D'après le renvoi à $B H G$ I 469, p. 65, n² 24, il doit s'agir d'une version prémétaphrastique.

${ }^{35} P G 87,3$, col. 3677-3680.

${ }^{36} P G 87,3$, col. 3685-3688. Cet ermite aurait apporté à l'empereur Théodose I une assistance surnaturelle dans sa lutte contre les "Barbares " d'Occident. L'histoire, répétée par Michel Glykas, Annal., p. 478, est visiblement calquée sur celle de Jean de Lycopolis dont le rôle auprès de Théodose a un fondement historique (voir C. Zuckerman, "The Hapless Recruit Psois and the Mighty Anchorite, Apa John ", Bulletin of the American Society of Papyrologists 32, 1995, p. 183-194).
} 
de Cyrille (384/412) ${ }^{37}$ est à première vue hors sujet. La biographie des saints s'écarte de V1, surtout celle de Jean, en qui V2 voit un Oriental, originaire d'Édesse selon $\mathrm{G}, \mathrm{M}$ et Met. Il séjournait à Jérusalem, lorsqu'il entendit parler de $\mathrm{Cyr}^{38}$. Avant de se tourner vers l'ascétisme en un village $(\kappa \omega ́ \mu \eta)$ de la côte arabique de l'Égypte,

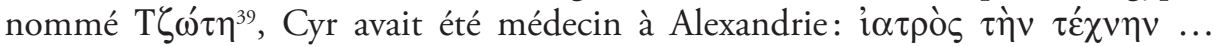

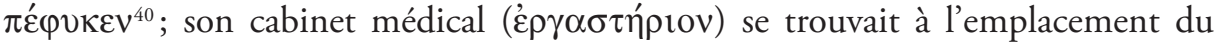
martyrium des Trois-Enfants, au lieu-dit $\Delta \mathrm{o} \rho v^{\prime} \zeta \mathrm{t} \mathrm{v}^{41}$.

Du point de vue liturgique, V2 offre l'avantage de donner une date pour la translation (28 juin), alors que V1 et Sophrone n'ont que celle des exécutions. Ainsi s'explique que C et L contiennent deux Vies des mêmes saints ${ }^{42}$. Dans un état originel, dont le Synaxaire de Constantinople a conservé le reflet, V2 ne mentionnait pas Ménouthis et Cyrille et s'arrêtait à la découverte des corps, qu'elle plaçait bien au 28 juin, mais entre 395 et 408, sous l'évêque Théophile. Cette question est cruciale pour l'histoire du culte de Cyr et Jean et je la traite en détail plus bas.

Postérieure au pontificat de l'évêque chalcédonien d'Alexandrie Apollinaire (551/570), qu'elle mentionne avec faveur ${ }^{43}$, V2 semble avoir subi, entre 615 et le $\mathrm{IX}^{\mathrm{e}}$ s. (L), des remaniements destinés à l'adapter au style et aux vues de Sophrone, et même à mettre dans la tête du public que Sophrone en était bien l'auteur. Du moins V2 suppose-t-elle connue son œuvre, car elle comporte parfois ses clausules rythmiques, qui sont à l'origine, semble-t-il, des divisions de $\mathrm{E}^{44}$. Son intitulé dans

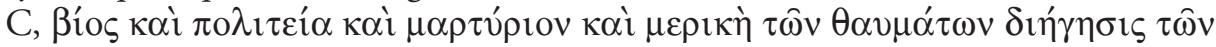

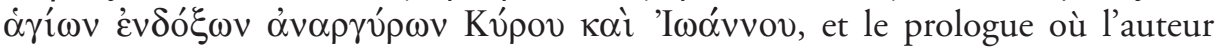
expose ses desseins montrent, comme l'a vu Nissen, que V2 était normalement suivie d'un choix de miracles ${ }^{45}$, ce qui est confirmé par F qui, à la suite de V2, a 15 miracles, et par G qui en conserve 9. Or il s'agit de pièces sophroniennes, quelque peu récrites dans F. Sa partie finale (la translation à Ménouthis), que je ne considère pas comme originelle, n'est qu'un résumé très sec du Panégyrique.

${ }^{37} P G 87,3$, col. 3688.

${ }^{38} P G 87,3$, col. 3681 .

${ }^{39} P G 87,3$, col. 3681 .

${ }^{40} P G 87,3$, col. 3677 et 3680 .

${ }^{41}$ C'est dans M, Met. (PG 114, col. 1233) et F qu'apparaît ce toponyme, disparu dans la lacune de V2 dans C, mais L l'avait déjà, encore qu'il se soit mal transmis (apud Orizin, AB 8, 1889, p. 96).

${ }^{42}$ Sauf dans l'exemplaire du ms. Kutlumusiu 37, V1 utilise le calendrier égyptien avec l'équivalence latine (6 mecheir, 31 janvier, col. 3692), alors que V2 se contente du calendrier latin, ce qui suggère que, dans son état présent, elle a été adaptée à la liturgie d'un sanctuaire non-égyptien.

${ }^{43}$ Ce parti pris explique qu'elle n'ait pas circulé dans l'Égypte médiévale.

${ }^{44}$ Deux exceptions: il s'agit du $\$ 9, P G 87,3$, col. 3684, où le texte grec, au vu de $\mathrm{L}$, pourrait avoir été remanié, et du $\$ 11$, ibid., col. 3685, où l’anomalie pourrait résulter de l’interpolation finale de

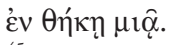

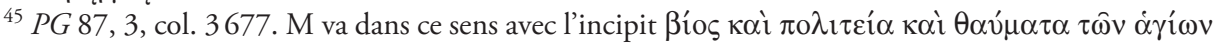

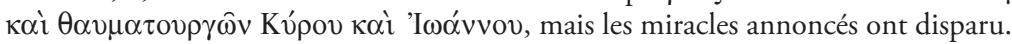




\subsection{Les Oratiunculae}

À la fin de sa composition, le narrateur de V2 annonce qu'il va donner en

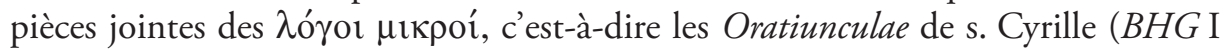
472-474; CPG III 5262), auxquelles Sophrone se réfère dans la Préface comme à sa source unique ${ }^{46}$. Cette annexe a été déplacée dans $C$, où les Oratiunculae sont enclavées entre les deux Vies, mais L, $\mathrm{f}^{\mathrm{o}}$ 38-40, (BHL nov. suppl. 2077b-d) les a à l'endroit attendu ${ }^{47}$. On en compte trois, alors que Sophrone et la version L de V2 n'en connaissaient que deux ${ }^{48}$. Après leur édition par Mai, elles ont été intégrées aux ouvres de Cyrille dans PG 77, col. 1100-1105. Je les appelle Or. I (col. 1 100), Or. II (col. 1 101-1 104) et Or. III (col. 1 104-1 105).

Elles sont munies d'inscriptiones confirmant l'attribution à Cyrille, et datées successivement des 2, 7 et 8 epeiph, c'est-à-dire qu'elles encadrent le jour de la translation donné par V2, 28 juin, ou, en termes égyptiens, 4 epeiph ${ }^{49}$. Les voici telles que je les ai trouvées dans $\mathrm{C}$ (en corrigeant les phonétismes), car Mai les a modifiées, comme on le verra dans les notes.

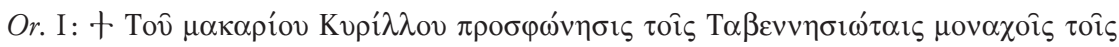

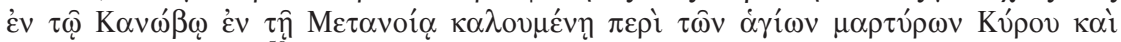

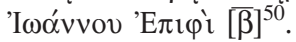

«Du bienheureux Cyrille, allocution aux moines tabennésiotes de Canope, en ce qu'on nomme la Métanoia, sur les saints martyrs Cyr et Jean, le deux epiphi ».

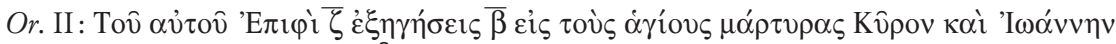

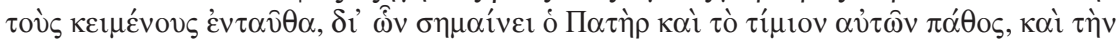

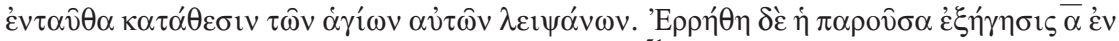

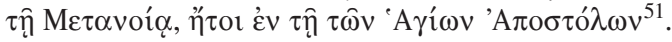

"Du même, le 7 epiphi, deux exégèses en l'honneur des saints martyrs Cyr et Jean qui gisent ici, par lesquelles le Père décrit leur précieuse Passion ainsi que l'inhumation ici de leurs

${ }^{46}$ Bringel, $\$ 1$.

${ }^{47} \mathrm{G}$ a la dernière des trois ( $\left.\mathrm{f}^{\mathrm{b}} 325^{\mathrm{r}}-326^{\mathrm{r}}\right)$, mais sous l'inscriptio d'Or. I, avec une date julienne "refaite " du 28 juin, au lieu du 2 epeiph (26 juin).

48 Panégyrique, Bringel, $\$ 1 ; P G 87,3$, col. 3690.

${ }^{49} \mathrm{~L}$, qui reproduit correctement les dates égyptiennes, donne des équivalences juliennes en retard d'un mois, peut-être parce que la commémoraison romaine de nos saints se célébrait alors à la fin de juillet.

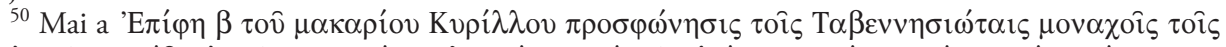

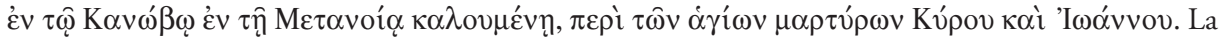
restitution du quantième du mois se tire de $\mathrm{L}$.

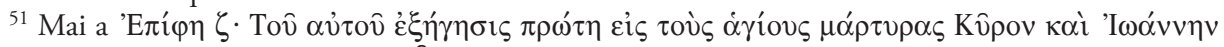

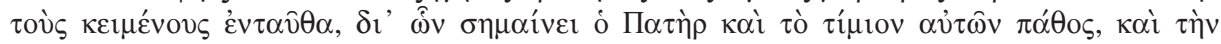

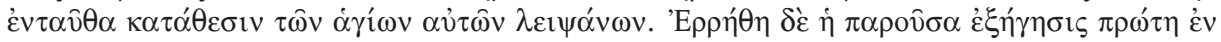

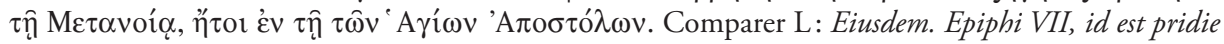
Kalendarum Augustarum relationes duc in sanctos martyres abba Cyrum et Joannem, qui hic requiescunt; per quas hic Pater insinuat et honorabilem eorum passionem, et depositionem sanctarum reliquiarum. Dicta est autem prosens prima narratio in Metance, quod interpretatur Ponitentia, sive in ecclesia Sanctorum Apostolorum. 
saintes reliques. La présente première exégèse fut prononcée à la Métanoia, c'est-à-dire en l'(église) des Saints-Apôtres ».

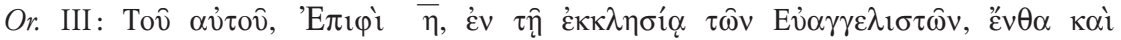

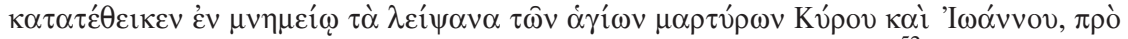

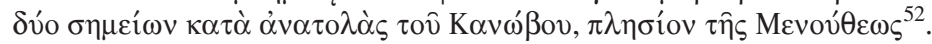

«Du même, le 8 epiphi, en l'église des Évangélistes, où il avait aussi inhumé dans un tombeau les reliques des saints martyrs Cyr et Jean, à deux milles à l'est de Canope, près de Ménouthis ".

Dans Or. I, très courte, l'auteur exalte les vertus de courage et de patience propres aux saints en général.

Dans Or. II, censée postérieure à la translation, l'auteur " répète " à son public ce qu'il a fait à cette occasion. Il justifie par la maladie sa brièveté, le manque de nouveauté du sujet et sans doute le manque d'art du présent discours. En voici la traduction complète:

"Nous n'avons pas été pris de paresse pour notre sermon habituel, mais, comme l'a dit le Sauveur: "Certes, l'esprit est ardent, mais la chair est faible" ${ }^{53}$. Nous avons toutefois confiance que par vos prières, avec la miséricorde de Dieu, notre état s'améliorera. Quant à ce que nous avons décidé et accompli pour l'utilité, nous avons considéré qu'il était nécessaire de vous le répéter.

Donc, vu que ce district manquait de médecins guérissant par Dieu, afin de rendre service à toute la région et au premier chef à celle qui environne l'église des Saints-Évangélistes (car le public, qui n'avait pas de martyrium ${ }^{54}$, s'en allait en certains autres lieux et, encore que chrétien, chutait), nous fûmes, pour cette raison, contraints de rechercher des reliques de saints martyrs. De fait, nous découvrîmes et apprîmes avec certitude qu'à l'époque où subissaient le martyre les vierges saintes dont nous avons le divin puits ${ }^{55}$ chez le saint évangéliste Marc, il y avait deux personnes dont l'une était un moine qui pratiquait l'ascèse, et l'autre un soldat, qui les assistaient pour les stimuler et les entraîner à la lutte athlétique, en sorte qu'elles soutinssent avec résolution le danger pour notre Sauveur. Eux aussi ont été appelés avec elles au martyre. Ils s'y rendirent vaillamment, ont témoigné pour le Christ et se sont pour lui défaits de leurs âmes. Les reliques des saints martyrs gisaient donc ensemble en un tombeau unique, et comme les reliques étaient indistinctes, car il n'était pas facile de distinguer qui était l'un et qui était l'autre, nous fûmes contraints de prendre ensemble les deux corps, et nous les transférâmes et déposâmes en l'église des Évangélistes, après avoir élevé le tombeau d'usage pour des martyrs. Réunissons-nous donc demain, si Dieu veut, pour honorer ensemble les saints évangélistes et les bienheureux martyrs. Ils s’appellent Cyr et Jean ”.

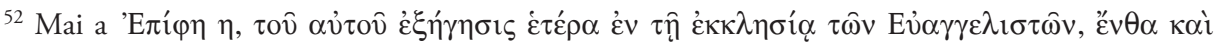

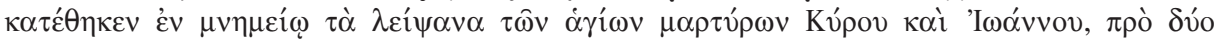

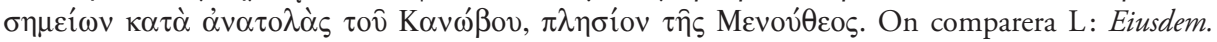
Epiphi VIII, id est Kalendas Augustas apud ecclesiam Evangelistarum, ubi in monumento recondidit reliquias sanctorum Cyri et Joannis, a duobus millibus de parte orientis Canopi juxta Menutheos.

${ }^{53}$ Mt. 26, 41; Mc. 14, 38.

${ }^{54} \mu \alpha \rho \tau$ prov; L a oraculum.

${ }^{55} \pi \eta \gamma \eta$ signifie plutôt puits que fontaine ou source, si on a à l'esprit les réalités égyptiennes. 
Or. III intéresse beaucoup les historiens des religions, car elle semble décrire, ce qui n'arrive pas si souvent ${ }^{56}$, la substitution d'un culte médical chrétien à un oracle païen. En voici la partie finale:

«Ils (Cyr et Jean) ont accompli le précepte en prenant eux aussi la croix et suivant leur Maittre. Ils n'étaient pas seuls à être conduits aux combats, mais il y avait aussi un chœur de vierges saintes qui, encore qu'elles fussent des femmes, étaient d'une détermination inébranlable. Avec elles, finit aussi sa vie cette belle paire d'athlètes. Comme salaire de leur amour pour le Christ, ils reçoivent le pouvoir de fouler aux pieds Satan et chasser les méchants démons. Que se présentent donc ceux qui s'égaraient autrefois, qu'ils viennent $a^{57}$ dispensaire authentique et probe, car personne ne nous forge des présages oniriques, personne ne dit aux visiteurs: "La Dame a dit: "Fais ceci ou cela". Veut-elle être en même temps une dame et un dieu puissant, et être adorée $?^{58}$ Alors que chez les démons, il n'y a ni mâle ni femelle, voyez quel est leur dessein, pour vouloir être appelés de noms de femmes. Après avoir donc piétiné les fables de vieilles et les charlataneries obsolètes des sorciers, qu'ils aillent trouver les authentiques et célestes médecins que Dieu tout-puissant a gratifiés du pouvoir de guérir, selon ses mots: "Guérissez les malades: vous avez reçu gratis, donnez gratis" 59 . Après donc avoir profité de tous les soins qui viennent du Sauveur, ils chantent ${ }^{60}$ leur Seigneur afin de mériter aussi le royaume des cieux [doxologie]».

Les trois Oratiunculae ont eu le même public: les moines du célèbre monastère pachômien (ou tabennésiote) de la Métanoia ${ }^{61}$. Leur établissement de Canope (plus précisément, selon Or. II, leur église des Saints-Apôtres) fut le cadre des deux premières. La troisième eut pour occasion une synaxe en l'église des Évangélistes, près de Ménouthis, à laquelle ces moines, selon la fin d'Or. II, avaient été invités.

Néanmoins, on distingue dans ce montage unitaire (forcé par Mai, avec sa manière de placer les dates au début des intitulés) deux strates mal combinées. Au temps de Sophrone, on ne lisait que les deux " exégèses " annoncées par l'inscriptio d'Or. II, c'est-à-dire Or. II elle-même, et sans doute Or. III. Elles ne devaient pas porter de date, sinon Sophrone en aurait tiré parti dans son Panégyrique. Plus tard (et donc après 615) s'accrédita la date du 4 epeiph/28 juin (connue, je le rappelle, de V2, mais ignorée de Sophrone), et l'on trouva utile de faire entrer les exégèses dans le calendrier des fêtes de Ménouthis. C'est alors que l'on découvrit Or. I, ou plutôt que l'on eut besoin d'elle pour fixer, au moins approximativement, l'anniversaire de la translation. Comme on l'a vu, Or. II est postérieure à cet événement fondateur et fut datée en conséquence. Mais Or. I ne pouvait être attribuée directement au 4 epeiph, sinon Or. II perdait son utilité, n'étant plus en effet qu'une simple " répétition ». Peu importait qu’Or. I n’eût aucun contenu, dès lors qu’on y trouvait

\footnotetext{
${ }^{56}$ P. Maraval, Le christianisme de Constantin à la conquête arabe, Paris, 1997, p. 46, parle à ce propos d' " un exemple presque trop clair (et qui en ce sens reste unique) ». Je serais tenté de commenter: testis unus, testis nullus.

${ }^{57}$ Mai a omis $\tau$ ó.

58 " être adorée ", $s c$. en tant que dieu masculin et féminin.

${ }^{59}$ Citation partielle de Mt. 10, 8.

${ }^{60} \mathrm{Au}$ lieu de la $3^{\mathrm{e}}$ pers. de l'ind., L, plus logiquement peut-être, a collaudemus et plus loin mereamur.

${ }^{61}$ Voir ci-dessous, n. 150.
} 
un titre et une date de peu antérieure au 4 epeiph. Les inscriptiones furent donc datées, mais les commanditaires de ces altérations ne se soucièrent pas de cohérence puisqu'ils laissèrent dans $O r$. II, après l'insertion du 7 epeiph, l'essentiel de l'ancien intitulé coiffant les deux exégèses originelles, si bien que les deux discours semblent annoncés le même jour ${ }^{62}$. On comprend donc mieux les inexactitudes éditoriales de Mai, qui, dans son esprit, devaient être des corrections légitimes. L'incohérence ne s'arrête pas là: alors que, d'après la dernière phrase d'Or. II, les noms de Cyr et Jean n'ont été proclamés que le 7 epeiph, $O r$. I a déjà ces martyrs pour sujet dès le 2 epeiph.

Je soupçonne aussi que les allusions aux moines de la Métanoia ne sont pas originelles, mais contemporaines de la phase de « découverte " d'Or. I et de datation corrélative de l'ensemble. En effet, Sophrone ne dit pas dans son Panégyrique devant quel public Cyrille s'est exprimé ${ }^{63}$. Son allusion à l'église des Apôtres ${ }^{64}$ est sans rapport avec les Oratiunculae. Comme il admirait les tabennésiotes ${ }^{65}$, son silence ne peut s'expliquer que par l'ignorance et non pas par une censure. Même

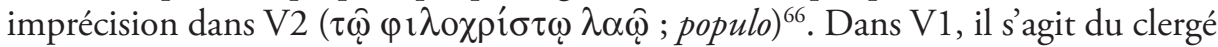

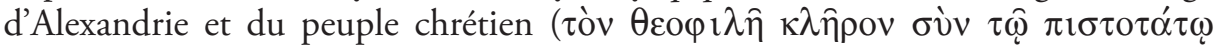
$\lambda \alpha \hat{\varphi})^{67}$. V1, qui n'en savait pas plus que Sophrone et V2, a simplement inventé un public plausible.

L'inscriptio d'Or. III présente une dernière anomalie. Puisque nous savons par $O r$. II que ces pièces étaient conservées à Ménouthis $(\hat{\varepsilon} v \tau \alpha \hat{v} \theta \alpha)$, quelle était l'utilité d'ajouter que le mausolée était à deux milles à l'est de Canope, près de Ménouthis? Cela n'apprenait rien à personne. On a l'impression que ceux qui ont voulu cette inscriptio cherchaient à imposer une équivalence toponomastique entre une église des Évangélistes et le tombeau de Ménouthis, équivalence qui peut-être n'allait pas de soi. Quant au sujet, l'extirpation de l'oracle de la Dame (à première vue,

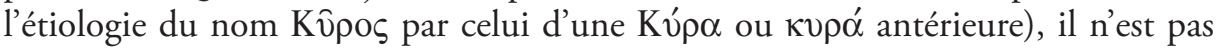
ce qu'annonçait l'intitulé d'Or. II, commun aux deux exégèses originelles. Dans les deux cas, Cyrille devait traiter du martyre et de la translation. Or. III est-elle la deuxième exégèse connue de Sophrone, ou bien un texte remanié ou substitué?

À l'aide du TLG_E, j'ai comparé les Oratiunculae au reste des œuvres de Cyrille. Or. I est trop courte pour qu'il en résulte rien de concluant. Or. II semble au-

\footnotetext{
${ }^{62}$ Le nom de Cyrille (

${ }^{63}$ Bringel, $\$ 27$.

${ }^{64}$ Bringel, $\$ 14$.

${ }^{65}$ Miracle $n^{\circ} 46 ₫ 4$.

${ }^{66} P G 87$, 3, col. 3689-3690.

${ }^{67} P G 87,3$, col. 3696.
} 
dessous de l'art de Cyrille ${ }^{68}$. En revanche, Or. III porte la marque incontestable du vocabulaire, du style ${ }^{69}$ et même de la pensée ${ }^{70}$ de Cyrille. La " création » de Cyr et Jean s'accorderait bien avec une action authentique de Cyrille: selon l'historien Socrate, il aurait promu en 415 à la dignité de martyr, sous le nom de Thaumasios, le moine téméraire Ammônios, torturé à mort par le gouverneur Oreste ${ }^{71}$.

Ces constatations devraient conduire à discuter une nouvelle fois l'authenticité cyrillienne des Oratiunculae, mise déjà en doute par $M^{\mathrm{gr}} \mathrm{L}$. Duchesne ${ }^{72}$. Ce savant ne se fondait pas sur un examen stylistique, mais alléguait des difficultés touchant au contexte historique. Malgré mon appréciation favorable d'Or. III, je crois que les arguments de Duchesne ont encore de la force et j'en ajouterai plus bas de nouveaux, mais l'analyse qui précède suffit à montrer que la collection des Oratiunculae présente des traces d'interventions tendancieuses, qu'elle est donc suspecte, si bien que je serais malgré tout enclin à voir, dans Or. III, un faux. Puisque nous savons que Cyrille a été falsifié ${ }^{73}$, pourquoi n'aurait-il pas été

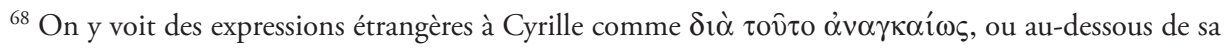

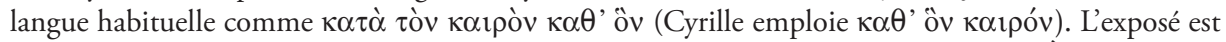
simple et narratif, sans effets: ce n'est pas le ton de l'" exégèse " cyrillienne habituelle. À la différence d'Or. III, il n'y a pas de doxologie finale.

${ }^{69} \mathrm{Or}$. III contient des mots ou expressions très prisés de Cyrille: $\varepsilon^{\prime} \mu \pi \alpha \imath \gamma \mu \alpha$ (charlatanerie de païens), $\dot{\alpha} \gamma \alpha \dot{\pi} \eta \sigma ı \varsigma$ (l'amour envers les parents), $\alpha v \tau \imath \mu \imath \sigma \theta i ́ \alpha$ (récompense), $\gamma \rho \alpha \omega ́ \delta \eta \mu \nu \theta \dot{\alpha} \rho \imath \alpha$, «contes de vieilles ", pour la mythologie païenne (7 attestations sur 10 dans le TLG_E, y compris la nôtre). Pour

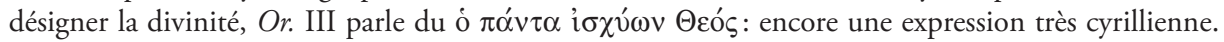
Une locution banale comme $\varphi \eta \mu$ i $\delta \eta$ revient 274 fois chez Cyrille sur les 1128 attestations procurées par le TLG_E. Encore qu'elle soit en faveur au Bas-Empire, aucun autre Père de l'Église ne l'emploie aussi souvent que lui. On pourrait déplacer l'analyse vers les citations scripturaires: ainsi le passage de

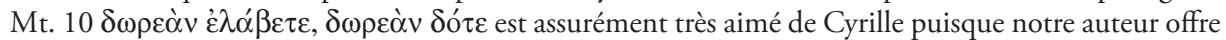
à lui seul plus du quart des citations enregistrées dans le TLG_E. Le ton d'ensemble, très oratoire, sinon exalté, s'accorde bien avec l'art de ce grand prédicateur.

${ }^{70}$ Voir J.-M. Sansterre, "Apparitions et miracles à Ménouthis: de l'incubation païenne à l'incubation chrétienne ", Apparitions et miracles, Bruxelles, 1991, p. 69-83 (ci-après Sansterre), notant, p. 72, que l'auteur, loin de se prononcer en faveur de l'incubation, se montre hostile aux oracles oniriques, ce qui recoupe les sorties de Cyrille contre la divination par les songes dans son Contre Julien: « Nous n'avons pas coutume de rechercher les visions par des songes, ni de nous réjouir puérilement à cause de vaines

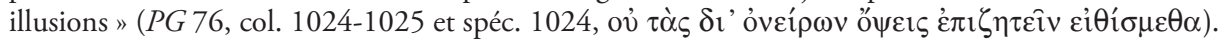
Du reste, l'auteur d'Or. III ne s'explique guère sur la médecine nouvelle qu'il entend instaurer. L'oracle de la Dame, qui implique l'intervention d'un interprète ou onirocrite qui décide si le songe est inspiré et quel sens il faut lui donner, n'annonce pas l'incubation chrétienne, car les visiteurs de Cyr et Jean déchiffraient eux-mêmes leurs rêves.

${ }^{71}$ Hist. eccl., 7, 14, Hansen, p. 360. Cette initiative ne fut pas approuvée par les chrétiens prudents d'Alexandrie si bien que le culte de Thaumasios périclita vite.

72 "Le sanctuaire d'Aboukir », Bulletin de la société archéologique d'Alexandrie 12, 1910, p. 3-14, spéc. p. 10-12 (ci-après Duchesne).

${ }^{73}$ Anastase le Sinaïte, Hodègos X, 2-7, Uthemann, p. 188-189: un gouverneur d'Alexandrie (Augustal)

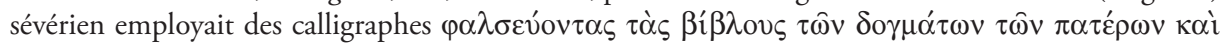

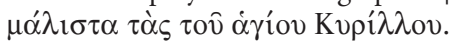


pastiché? Une autre hypothèse (et c'est à peu près celle de Duchesne) serait que l'écrit, émanant d'un auteur inavouable (ainsi un monophysite) et postérieur à Cyrille, mais imbu de ses œuvres, a été réattribué lorsque les chalcédoniens mirent la main sur le martyrium ${ }^{74}$. Quand bien même je me tromperais, ce qui importe, ce n'est pas que Cyrille ait promu des saints nommés Cyr et Jean, mais que ces derniers aient opéré à Ménouthis. Or, considérée sans sa curieuse inscriptio, Or. III ne comporte aucun toponyme, et donc rien qui puisse appuyer les prétentions de Ménouthis. Les précisions qui pourraient aller dans ce sens, la découverte des corps, leur réinhumation en l'église des Évangélistes, sont toutes dans Or. II, pièce d'authenticité bien moins évidente. De même, les personnalités hagiographique, cultuelle et iconographique des deux héros, Jean comme soldat, et Cyr comme

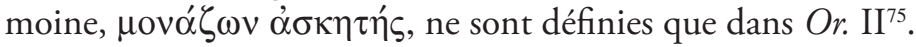

\subsection{Le corpus sophronien}

Cette œuvre monumentale ${ }^{76}$, que nous divisons, d'après Sophrone lui-même, en trois parties, Préface (BHG I 475; CPG III 7645 ; voir BHL I 2079), Panégyrique (BHG I 476; CPG III 7645 ; voir BHL I 2 079) et Miracles (BHG I 477-479; CPG III 7646 ; voir $B H L$ I 2080), formait dans l'esprit de l'auteur, un livre unique, dont la Préface était en quelque sorte l'introduction méthodologique ${ }^{77}$. C'était aussi l'opinion des Pères du II ${ }^{\mathrm{e}}$ concile œcuménique de Nicée (787), auxquels ont été soumis des extraits de cet ouvrage appuyant le culte des images ${ }^{78}$. P. Bringel, dans son édition de la Préface et du Panégyrique, défend la même vue: les Miracles concourent au Panégyrique. Ils font partie de l'éloge des saints, auquel ils ajoutent un " effet de vérité ». Cependant, d'après des manuscrits inconnus de Mai, le livre de Sophrone circulait aussi en morceaux choisis: nous avons ainsi, par M, G et le ms. Marcianus gr. 0595 (Mioni 583; ci-après V) ${ }^{79}$, des copies intégrales ou

\footnotetext{
${ }^{74}$ Duchesne cite une substitution analogue, celle du nom de Cyrille à celui de Théodore de Mopsueste dans les diptyques de cette cité.

${ }^{75}$ Cette manière de caractériser Cyr n'est pas un indice de haute antiquité de la légende. Voir Th. Baumeister, Martyr invictus, Münster, 1972, p. 22.

${ }^{76}$ Tout ce qu'a écrit Sophrone sur nos saints n'est pas dans C. Il faut tenir compte de deux de ses poèmes anacréontiques, les $\mathrm{n}^{\text {os }} \mathrm{XV}$ et XVI ( $P G 87,3$, col. 3800-3801; je n'ai pas pu me procurer l'édition et traduction de M. Gigante, Sophronii Anacreontica, Rome, 1957), le premier étant réduit à son inscriptio, le second très lacunaire et sans doute corrompu. Mais Matter a montré, p. 18-21, que le premier devait se rapporter à l'ophtalmie désespérée qui le conduisit au sanctuaire (miracle $\mathrm{n}^{\circ} 70$ ) et le second à une célébration de nos deux saints. Ces textes ne pouvaient pas remplir de fonction liturgique directe, mais de nombreux miraculés prenaient la peine de marquer leur reconnaissance aux saints en leur élevant des hymnes.

${ }^{77}$ Il y parle de ses sources, expose le plan des $\Theta \alpha v ́ \mu \alpha \tau \alpha$, son classement des miraculés et même ses principes d'écriture.

${ }^{78}$ Mansi XIII, p. 57-60.

${ }^{79} \mathrm{~V}$ est un ménologe prémétaphrastique de janvier d'origine athonite attribuable pour l'essentiel au début du XI ${ }^{\mathrm{es}}$ Il a, à la date du 31 janvier, le début et la fin du Panégyrique.
} 
partielles ${ }^{80}$ du Panégyrique; F, G, V et certains manuscrits arabes ont des sélections de miracles.

Les circonstances et la date de la composition sont connues. Peu avant 608, Sophrone se trouvait à Alexandrie, en compagnie de son ami et maître en ascèse, Jean Moschos. Il y séjourna jusqu’à leur départ pour Rome vers 615. Sophrone se lia alors aux évêques chalcédoniens Euloge (581/608) et Jean l'Aumônier ou de Chypre (610/619). Sous le dernier pontificat, et donc entre 610 et 615 (miracle $n^{\circ} 8$ ), il fut pris d'une maladie d'yeux dont il guérit à la suite d'un séjour à Ménouthis (miracle $\left.\mathrm{n}^{\circ} 70\right)$. Son livre fut le témoignage de sa reconnaissance, une sorte d'ex-voto dont l'auteur consacra un exemplaire au sanctuaire. Gratitude moins personnelle que militante, car Sophrone sert des causes, celle du chalcédonisme ${ }^{81}$, et celle, moins avouée mais plus intéressée, du clergé de Ménouthis, qui souhaitait imposer une image particulière de $\mathrm{Cyr}$ et Jean et ne laisser aucune chance à des sanctuaires concurrents.

Sophrone admet dans la Préface que sa documentation était mince: «Étant allés voir, à cause d'une maladie d'yeux, Cyr et Jean les martyrs, nous séjournions dans leur temple. Considérant la masse des guérisons, nous voulûmes lire des documents nous enseignant le combat des martyrs et proclamant quelques-uns des miracles antérieurs. Nous ne trouvâmes rien de tel, à l'exception de deux petites homélies de Cyrille, ce grand champion et héraut de la vérité " ${ }^{82}$. À en juger d'après le Panégyrique, il ne faut pas le croire sur parole.

\subsubsection{Préface et Panégyrique}

Pour la tradition manuscrite, le lecteur est invité à se reporter à l'édition de P. Bringel.

Conforme aux préceptes du genre, d'une verbosité exaltée qui peut heurter le goût de notre temps, cette pièce, qui combine biographie, récit de passion et éloge, est riche en données historiques et cultuelles. Les Oratiunculae ont procuré les thèmes fondamentaux: Cyr était moine, Jean, soldat; ils ont subi le martyre en compagnie de femmes qu’ils assistaient; leurs reliques mêlées ont été transférées par Cyrille en l'église des Évangélistes; ces corps indistincts forment une dyade puissante et bienfaisante; ils ont vaincu l'oracle local d'un démon féminin qui dupait jusqu'aux chrétiens; ils soignent gratuitement ceux qui viennent les voir. L'auteur a enrichi ce canevas de détails et expressions où il se montre en bon accord avec V1. Je relève les convergences historiques les plus frappantes. Compensant une déficience cultuelle des Oratiunculae, les deux écrits ont la date des exécutions, exprimée à la fois en termes égyptiens et juliens. Ils la placent tous deux après la mort de l'évêque Pierre d'Alexandrie ${ }^{83}$. Alors que les Oratiunculae ne disent rien

\footnotetext{
${ }^{80}$ Se reporter à l'introduction de Bringel.

${ }^{81}$ Voir sur ce point Flusin II, p. 65.

${ }^{82}$ Bringel, $\$ 1$.

${ }^{83}$ Bringel, $\$ 18$ et $23 ; P G 87,3$, col. 3692.
} 
là-dessus, ou ne parlent que d'un point d'eau lié au souvenir des compagnes des saints, Sophrone sait avec $V 1^{84}$ que les reliques ont été déposées puis redécouvertes à Saint-Marc. Le nom du démon Ménouthè, éponyme de Ménouthis, n’est pas dans les Oratiunculae, mais est connu des deux écrits ${ }^{85}$, et de même ceux des vierges, de Kasios, le prêtre païen de Canope, et du gouverneur Syrianos ${ }^{86}$. Sur le plan de l'expression, les deux écrits se recoupent fréquemment, ainsi dans les récits du martyre et de la ruine du sanctuaire de Ménouthis, disparu sous le sable et la mer ${ }^{87}$. Certaines transitions sont à peu près identiques ${ }^{88}$. Mais Sophrone et V1 peuvent diverger: ainsi les vierges, dont les deux écrits placent l'origo à Canope (rien dans les Oratiunculae), ne sont pas nommées dans le même ordre: alors que V1 a la séquence Théodote, Théoctiste, Eudoxie, chez Sophrone (suivi par V2), Théoctiste précède Théodote. Fidèle aux Oratiunculae, Sophrone reconnaît que le démon attirait des chrétiens ${ }^{89}$. Le ms. Lavra 327 de V1 est seul à enregistrer cette tradition peu édifiante ( $\tau$ òv $\lambda \alpha o$ ó). Elle a été censurée dans les autres exemplaires, au mépris de la syntaxe dans $\mathrm{C}^{90}$. Je suis encore tenté d'attribuer à une censure la disparition complète du nom de Théophile dans V1, alors que Sophrone rappelle qu'il fut le prédécesseur méritoire de Cyrille ${ }^{91}$, et célèbre en lui le fondateur de l'église des Évangélistes (silence des Oratiunculae). Le récit du rêve de Cyrille, où un ange lui révèle le lieu de repos des reliques à Saint-Marc, se présente différemment chez Sophrone et dans V1. Selon le Panégyrique, l'ange n’a parlé que du seul Cyr, alors que dans V1, il mentionne les deux saints ${ }^{92}$. La version de Sophrone se retrouve dans $\mathrm{V}^{93}$, mais elle était dangereuse (j’y reviendrai), et fut récrite dans V1. V1 fait bien allusion aux Oratiunculae, mais Cyrille les aurait prononcées avant ${ }^{94}$ la translation et non pas après, comme le veut le Panégyrique ${ }^{95}$ et l'indiquent leurs

\footnotetext{
${ }^{84}$ Bringel, $\$ 3,14,23,27 ; P G 87,3$, col. 3693, 3696.

85 Bringel, $\$ 24 ; P G 87,3$, col. 3693.

${ }^{86}$ Bringel, $\$ 18$; pour V1 (acéphale dans $C$ ), on se réfere aux autres manuscrits.

${ }^{87}$ Bringel, $\$ 29 ; P G 87,3$, col. 3696.

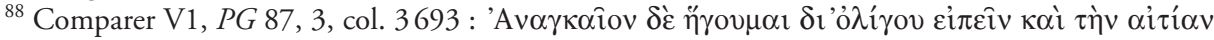

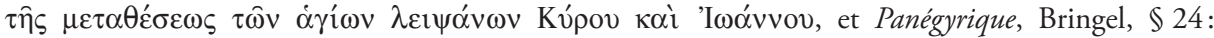

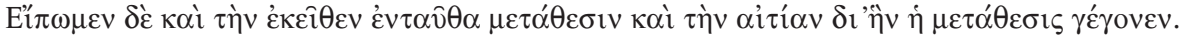

${ }^{89}$ Panégyrique, Bringel, $\$ 25$.

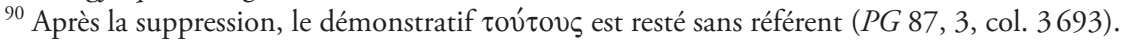

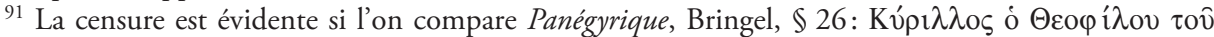

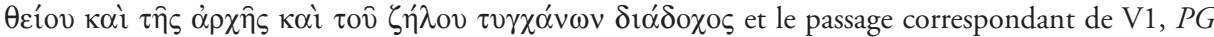

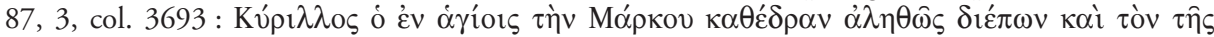

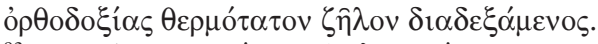

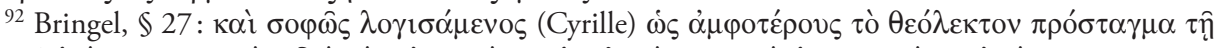

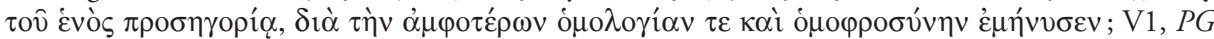

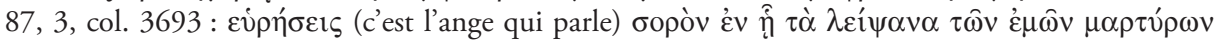

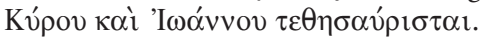

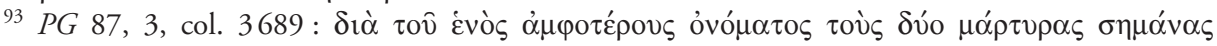
(l'ange).

${ }^{94} P G$ 87, 3, col. 3696.

${ }^{95}$ Bringel, $₫ 27$, en accord avec V2, $P G 87,3$, col. 3677 et 3681 .
} 
inscriptiones. Sophrone ne dit pas devant quel public Cyrille a pris la parole, mais selon V1, les exégèses s'adressaient au clergé et aux laïcs. Parfois, V1 en dit plus que Sophrone. Ainsi Cyr et Jean habitaient à Canope quand ils furent arrêtés ${ }^{96}$, mais Sophrone ne sait rien sur leur domicile.

Concordances nombreuses donc entre V1 et le Panégyrique, mais désaccords sur des détails qui, après examen, ne sont pas tous négligeables. Cela pourrait s'expliquer si l'on postulait une première forme de V1, accessible à Sophrone, mais dépouillée ensuite de traits devenus inacceptables pour la foi et le culte de Ménouthis.

Quant à V2, Sophrone ne l'a sans doute connue que dans l'état où la résume le Synaxaire de Constantinople, qui est incompatible avec les prétentions de Ménouthis et ses propres convictions ${ }^{97}$. Il s'en est servi néanmoins, car je ne vois pas dans quel autre écrit il aurait trouvé la légende faisant de Cyr un ancien médecin, qui n'a aucun appui dans V1 et les Oratiunculae ${ }^{98}$.

\subsubsection{Les Miracles}

Cette collection de 70 récits n'est conservée que par C et $\mathrm{L}, \mathrm{f}^{\circ} 61^{\mathrm{v}}-195^{\mathrm{v}}(B H L \mathrm{I}$ 2080). Sophrone n'y voyait qu'un mince aperçu des prodiges opérés par ses héros. De fait, comme nous l'avons vu, le ms. Kutlumusiu 37 de V1 enregistre aux fo 266 ${ }^{\text {- }}$ $269^{\mathrm{v}}$, cinq miracles inédits de Cyr et Jean (BHG I 479i ${ }^{99}$. Nous en avons encore en copte et en arabe. Sophrone nous apprend au miracle $n^{\circ} 30 \$ 13-14$ qu'il n'a pas tenu compte de récits qui circulaient aussi sous les noms d'autres saints de l'époque. Une sélection donc, mais imposante, sans parallèle et, vu la longueur de certaines pièces, mal adaptée à la lecture publique. Ainsi s'expliquent les anthologies de F, G et $\mathrm{V}^{100}$.

J'en propose à la suite de cette étude une traduction commentée.

\subsection{La tradition copto-arabe}

De méritoires recherches de Ramez Boutros auprès d'institutions religieuses égyptiennes ont révélé une tradition hagiographique copte et arabe sur nos saints illustrée par plusieurs icônes modernes, mais d'un type manifestement très ancien, par des manuscrits médiévaux et modernes, coptes et arabes, en particulier des

\footnotetext{
${ }^{96}$ PG 87, 3, col. 3689.

${ }^{97}$ Voir ci-dessous notre discussion de la " tradition cyrillienne ".

${ }^{98}$ Bringel, $\$ 11 ; P G 87,3$, col. 3677 et 3680 . À la différence de V2, Sophrone évite toutefois le terme

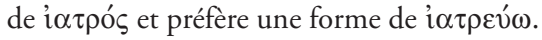

${ }^{99}$ Le mauvais état du manuscrit dans ses parties supérieure et médiane rend difficile une édition complète. Ces pièces sont d'ambiance alexandrine, encore que pauvres en données historiques. Du point de vue cultuel, elles sont en accord avec l'orthodoxie de V1.

${ }^{100} \mathrm{~V}$, aux fo $338^{\mathrm{v}}-341^{\mathrm{v}}$, n'a que les $\mathrm{n}^{\text {os }} 1$ et 23 (ce dernier sous forme incomplète), G, aux $\mathrm{f}^{\mathrm{o}} 341^{\mathrm{v}}-351^{\mathrm{r}}$, les $\mathrm{n}^{\text {os }} 1-4,7$ (cette pièce étant réduite à son inscriptio), 14, 15, 23 et 25, F, fo 214-237v , les $\mathrm{n}^{\text {os }} 1-7,23$, $26,27,41,42,45,46$ et 59 . Une sorte de paresse fait que l'on a puisé de préférence au début de la collection (les pièces 1-7 sont en général assez brèves). Le $\mathrm{n}^{\circ} 23$ est particulièrement inepte, mais fort apte à ébahir le public. Il figure dans toutes les collections et est aussi connu en version arabe.
} 
versions d'une Passion proche de V1, des hymnes, et des miracles dont une partie n'est pas sophronienne. Dans l'une de ces pièces, Cyr agit seul et à Saint-Marc. R. Boutros a donné un aperçu de ses découvertes le 9 novembre 2002 dans une table ronde sur Alexandrie médiévale organisée par C. Décobert et J.-Y. Empereur. Elles ne manqueront pas, quand toutes les pièces du dossier arabe seront publiées, de renouveler maints problèmes littéraires et historiques. Du moins est-il à présent acquis, malgré ce qu'on a longtemps pensé, que ce culte s'est maintenu sans interruption en Égypte jusqu’à nos jours.

\section{Que vaut le consensus?}

Si l'on a suivi notre discussion des sources, peu de points de ce que j'appelais plus haut le "consensus" devraient résister à l'examen. Voici dès à présent, en attendant des études particulières, un sommaire des résultats acquis.

\subsection{Les personnalités de Cyr et Jean}

On peut tout d'abord affirmer, non seulement que l'on connaît "peu de choses " sur Cyr et Jean, mais encore qu'il s'agit de martyrs apocryphes. Certains le savaient au temps de Sophrone (miracle $\left.n^{\circ} 29\right)$. Les fabulations de leurs Vies s'opposent à leur historicité, et principalement la date de leur martyre, le 31 janvier 312, sous "Dioclétien» et "Syrianos »"101. À cette époque, la persécution avait cessé en Égypte ${ }^{102}$. Le choix d'une telle date est bien à la mesure des prétentions du sanctuaire de Ménouthis: il s’agit de déposséder l'illustre évêque, et martyr authentique, Pierre I Ir d'Alexandrie, exécuté le 25 novembre 311, de son titre de sceau ou fin des martyrs, $\tau \dot{\varepsilon} \lambda{ }_{0} \varsigma \alpha \rho \tau \tau$ vo $\omega v$, très cher à l'Église d'Égypte pour des raisons symboliques et idéologiques ${ }^{103}$.

Bien que Cyr et Jean forment, à l'exemple de Côme et Damien, un couple hagiographique ${ }^{104}$, il y a lieu de penser que, dans cette paire, Jean n'est qu'un

${ }^{101}$ Il n'y a pas lieu de s'arrêter à la mention de Dioclétien, qui ne régnait plus en 312. Syrianos fut en réalité à Alexandrie, en 356, sous le titre de duc, l'agent haï de la politique arienne de Constance II (PLRE I, Syrianus). On comparera le gouverneur Sebastianos du Martyre d'Apa Epima, Mina, p. 29, 31,32 , homonyme d'un duc persécuteur des nicéens à la même époque (PLRE I Sebastianus 2).

${ }^{102}$ Le témoignage essentiel est ici la Vie de s. Antoine 47, 2, Bartelink, p. 362.

${ }^{103}$ Voir A. Martin, Athanase d'Alexandrie et l'Église d'Égypte au IV siècle (328-373), Rome, 1996, p. 248 et n. 96. Sophrone endosse cette donnée de V1 (PG 87, 3, col. 3692) dans le Panégyrique (Bringel, $\$ 18$ ), mais sous une forme atténuée, en mettant à bonne distance, dans son texte, l'allusion à Pierre et la date du 6 mecheir (Bringel, $\$ 23$ ). Noter que Sophrone ne reproduit pas une comparaison de V1 entre l'évêque martyr et l'apôtre Pierre, assortie d'une formulation favorable à la primauté romaine.

${ }^{104}$ Couple asymétrique, à l'avantage de Cyr. Cette inégalité est classique dans les dyades ou triades de saints (voir A. Papaconstantinou, Le culte des saints en Egypte, des Byzantins aux Abbassides, Paris, 2001, p. 131, à propos de Côme et Damien; ci-après Papaconstantinou, Culte des saints). 
personnage adventice, un Oriental connu ou honoré d'abord à Édesse, puis naturalisé égyptien dans des circonstances que nous ignorons. Cyr fut d'abord adoré seul. Dans des miracles non sophroniens, en grec ${ }^{105}$, en copte et en arabe, Cyr agit sans son compagnon, de même qu'il est seul sur une mosaïque du martyrium de Ménouthis (miracle $n^{\circ} 28 \$ 12$ ). Quand, selon le Panégyrique et V2, un ange révéla à Cyrille l'emplacement des reliques des saints, il ne prononça que le nom de Cyr. Il paraît que l'ange signifiait ainsi les deux saints sous un seul nom. Mettons que l'hagiographie a adapté au culte des deux saints un récit qui, à l'origine, ne concernait que le seul Cyr. On comprend que V1 n'ait pas voulu endosser une telle tradition. Le fait que Jean fut d'abord soldat est admis par toutes les sources (non sans variantes), mais la vision d'un Cyr médecin puis moine, proposée par le P. Delehaye, procède d'un compromis entre des légendes parallèles. Le clergé de Ménouthis n'avait pas intérêt à populariser cette vue profane de Cyr. Or. II, V1 et Sophrone mettent donc l'accent sur sa personnalité ascétique et V1 en fait un habitant du centre monastique de Canope, dans le voisinage immédiat du martyrium, et non pas de la ville d'Alexandrie.

\subsection{L'Isis de Ménouthis et les saints Cyr et Jean}

Le P. Delehaye parle d'Isis. S’il est vrai qu'une Isis fut adorée à Ménouthis à l'époque romaine ${ }^{106}$, une Isis dont la vocation oraculaire est bien marquée par l'épiclèse de "Vérité ", $\alpha \lambda \eta \dot{\eta} \theta(\varepsilon) \downarrow \alpha v$, que lui décerne une hymne sur papyrus ${ }^{107}$, l'hagiographie de Cyr et Jean ne connaît que la Dame d'Or. III ou le démon Ménouthè des Vies et de Sophrone. Ce nom, dont le lien avec Isis était perdu de vue depuis des siècles, procurait aux habitants du lieu l'explication du nom de leur village, de même que les mésaventures de cet être diabolique détrôné par les saints ajoutaient une strate triomphale à l'histoire mythique du martyrium. En effet, V1 prétendait, ce qui est plus facile à imaginer qu'à prouver, qu'avec l'installation des reliques, le temple avait disparu sous le sable d'une dune côtière et dans la mer:

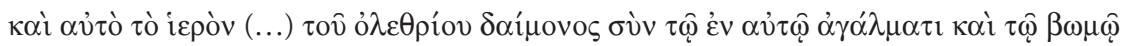

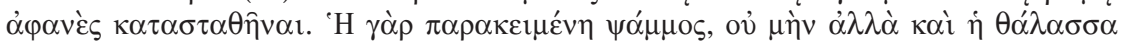
$\tau \hat{~}$ $\dot{\varepsilon} \pi \iota \lambda u^{\prime} \zeta o v \sigma \alpha, \dot{\eta} \delta \dot{\varepsilon} \kappa \alpha \tau \alpha \chi \omega ́ \sigma \alpha \sigma \alpha^{108}$.

Sophrone est du même avis dans le Panégyrique:

\footnotetext{
${ }^{105}$ Miracle n $^{\circ} 18$ d'Anastase le Perse, Flusin I, p. 153 (Jean est remplacé par Anastase).

${ }^{106}$ Les pièces à conviction sont deux dédicaces d'époque impériale de Porto, mentionnant une Isis de Ménouthis èv Mevoú日r (A. Bernand, Le Delta égyptien d’après les textes grecs I, Le Caire, 1970, p. 297, et Iscr. Gr. It., Porto, ${ }^{\circ} 18$, avec association avec Sérapis). L'essentiel de ce qu'on sait sur l'Isis de Ménouthis est rassemblé par F. Kayser, "À propos des cultes de Canope ", BIFAO 91, 1991, p. 214-217.

${ }^{107}$ P. Oxy. XI 1380, 63 (Pack² 2477); voir Montserrat, p. 258.

${ }^{108}$ PG 87, 3, col. 3696.
} 


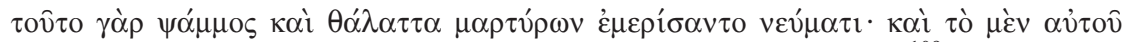

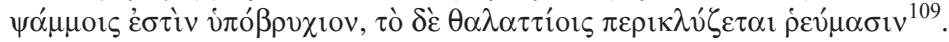

Au prologue du miracle $n^{\circ} 66$, il évoque:

«Le manteau du sable et de l'oubli, comme c'est le cas pour le démon figuré par Ménouthè, avec ses cérémonies, ses autels et son temple, dont l'oubli est bénéfique, selon le vœu des martyrs qui l'ont provoqué. Comme ce spectacle serait très dommageable aux curieux, il est dissimulé par le sable de la mer, qui en abolit entièrement le souvenir, y substituant l'utilité des choses invisibles. "

L'Isis de Ménouthis n'est mentionnée, à l'époque tardive, que par un écrit très antérieur au corpus hagiographique de Cyr et Jean, et sans rapport clair avec lui, la Vie de Sévère rédigée entre 512 et 518 par l'auteur monophysite Zacharie de Mytilène $^{110}$. Dans un récit célèbre qui se place vers $485 / 487$, sous le pontificat hérétique de Pierre Monge, l'auteur relate de la manière la plus vive et détaillée la destruction, avec le concours des moines tabennésiotes de Canope, d'un lieu de culte clandestin de la déesse. Malgré la rigueur des lois, cet oracle prospérait du fait de la tiédeur intéressée des chrétiens de l'endroit. Il y avait bien alors une église à Ménouthis, église dont Zacharie ne donne pas le vocable, mais son clergé corrompu tolérait les cérémonies rivales. Cette Isis, ou plutôt "le démon figuré par Isis ", agissait, comme Ménouthé ou la Dame d'Or. III, par des apparitions oniriques,

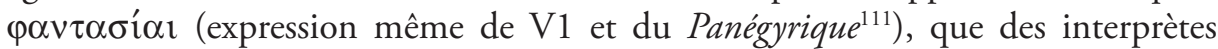
expliquaient ${ }^{112}$. L'auteur remarque que la déesse avait jadis à Ménouthis un temple que "quelqu'un a, accomplissant ainsi une bonne action, enfoui sous le sable (...), au point qu'on n'en voit même plus la trace $»^{113}$. Zacharie et les hagiographes de Cyr et Jean caractérisent Isis et Ménouthè en termes assez voisins. Ils ont aussi en commun l'image du temple disparu sous le sable. V1 et le chalcédonien Sophrone ne se seraient-ils pas inspirés de la Vie de Sévère, source pourtant inavouable? N'estce pas là l'explication de la réticence de V1 à nommer les "Pères " qui lui ont procuré sa description? Ou faut-il supposer que Zacharie et l'hagiographie de Cyr et Jean se font l'écho d'une légende alexandrine, qui, tout en constatant l'absence complète de vestiges païens à Ménouthis, voulait qu'un temple, désormais enfoui sous le sable, eût précédé les lieux de culte chrétiens?

Légende d'un genre au fond bien banal. À cette époque, comme l'a montré G. Dagron pour Constantinople, se développait autour des monuments chrétiens, dont l'histoire réelle était souvent perdue de vue, une littérature des origines, une branche du vieux genre rhétorique patriographique, tendant à les surimposer à

\footnotetext{
${ }^{109}$ Bringel, $\$ 29$.

${ }^{110}$ PO 2, p. 16-35.

${ }^{111} P G 87,3$, col. 3693 ; Bringel, $\$ 24,26$ et 29;

${ }_{112}^{112}$ PO 2, p. 18, 21.

${ }^{113} P O 2$, p. 19. On ne peut décider aisément si cette assertion se rapporte à un temple d'autrefois, antérieur à la proscription officielle des cultes païens à la fin $\mathrm{du}_{\mathrm{IV}} \mathrm{e}^{\mathrm{e}}$ siècle, ou bien au sanctuaire clandestin, qui fut lui aussi détruit de fond en comble par les activistes chrétiens (du moins si nous devons ajouter foi à ce récit, dont on évoque les anomalies à la fin de cette étude).
} 
des édifices païens antérieurs, comme pour mieux marquer leur antiquité et, en même temps que la menace latente des restes païens, l'expansion victorieuse du christianisme ${ }^{114}$. Il serait facile de montrer qu'une telle littérature a prospéré en Égypte et à Alexandrie au point d'empoisonner jusqu'à nos jours la recherche historique ${ }^{115}$.

\subsection{La valeur de la tradition cyrillienne}

Pour décrier les Oratiunculae, Duchesne remarquait qu'entre la fondation supposée de Cyrille et l'époque de Sophrone, nous n'avons aucune attestation du culte de Ménouthis. Il faisait notamment valoir le silence de la Vie de Sévère, qui, plusieurs décennies après l'œuvre cyrillienne, n'évoque ni l'église des Évangélistes, ni les saints Cyr et Jean, et trouve à Ménouthis un sanctuaire païen actif coexistant avec une église sans nom au clergé indigne. Delehaye a opposé à Duchesne ${ }^{116}$ que le silence n'est pas un argument et que le culte de Cyr et Jean a pu simplement piétiner entre Cyrille et le vir ${ }^{\text {s. }}$ Il reste néanmoins troublant que, depuis Duchesne, rien ne soit venu meubler ce vide documentaire. S. Timm allègue un écrit nestorien qui ferait mention du sanctuaire pour l'année 451, mais ce texte ose prêter à l'oracle des opinions nestoriennes, ce qui est impensable à l'époque. Au surplus, son éditeur expose de bonnes raisons d'attribuer ce passage à un ensemble interpolé, assignable au viI ${ }^{\mathrm{e}}$ s. ${ }^{117}$ Je vois aussi jointe au dossier une mosaïque de Gerasa, datée de 531, dont un panneau représenterait notre sanctuaire, à l'extérieur d'une ville entourée de murailles où certains veulent reconnaître Canope. En réalité, malgré sa proximité d'une figuration d'Alexandrie, ce panneau ne comporte pas de légende appuyant une telle identification ${ }^{118}$.

Duchesne aurait encore pu tirer argument du Synaxaire de Constantinople. Daté de 945/959, cet ouvrage comporte deux notices sur Cyr et Jean à leurs

${ }^{114}$ G. Dagron, Constantinople imaginaire, études sur le recueil des «Patria ", Paris, 1984, p. 91-92; voir pour l'ensemble de l'Orient, L. Foschia, «La réutilisation des sanctuaires païens par les chrétiens en Grèce continentale ", REG 113, 2000, p. 413-434, en particulier les p. 416-421.

115 Voir J. Gascou, "Les églises d'Alexandrie, questions de méthode ", Alexandrie médiévale 1, Études alexandrines 3, Le Caire, 1998, p. 23-44, plus spécialement p. 30-36, avec notamment la discussion du cas du Sérapéum; on peut placer sous ce jour la tradition apocryphe qui voit dans un temple de Kronos le monument qui aurait précédé l'église dédiée à saint Michel; voir p. 31 n. 51 (ci-après Gascou, Églises).

116 "Les saints d'Aboukir », $A B$ 30, 1911, p. 449 : «Il suffisait, semble-t-il, de dire que la cérémonie de la translation des reliques n'eut pas un effet foudroyant et qu'il fallut quelques années pour changer la face des choses » (ci-après Delehaye, Saints d'Aboukir).

117 F. Nau, Histoire de Nestorius d'après la lettre à Cosme, PO 13, 2, p. 281 (171). Ce texte est néanmoins cité par S. Timm, Das christlich-koptische Ägypten in arabischer Zeit I, "Bûqîr ", p. 442 (ci-après Timm).

${ }^{118}$ Voir Sansterre, p. 74, se référant au n 306 des inscriptions publiées par C. B. Welles, dans C. H. Kraeling, Gerasa (City of the Decapolis), New Haven, 1938, avec la pl. LXVII b. 
commémoraisons du 31 janvier (martyre) et du 28 juin (translation) ${ }^{119}$, solennités qui étaient célébrées en deux lieux de culte de la capitale, situés aux $\tau \alpha \grave{\Sigma} \Sigma \omega \rho \alpha \kappa i ́ o v$

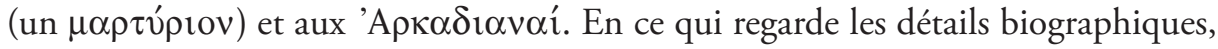
leur dépendance à l'égard de $\mathrm{V} 2$ est évidente: nous trouvons ainsi au 31 janvier que Cyr était alexandrin, que Jean venait d'Édesse. Fuyant la persécution, Cyr s'établit en un lieu proche de la mer Arabique où il prit l'habit monastique. Jean était à Jérusalem quand il fut attiré par la réputation de médecin de Cyr, qui guérissait "tout mal et toute faiblesse " ${ }^{120}$. Au 28 juin, Cyr et Jean ont les mêmes origines qu'au 31 janvier, à savoir Alexandrie et Édesse, la notice considérant que Cyr et Jean, et non plus le seul Cyr, étaient tous deux médecins ${ }^{121}$ soignant à nouveau toute

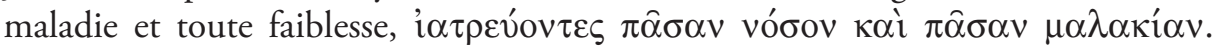
Mais alors que V1, Sophrone et, dans son état présent, V2 s'accordent pour placer l'affaire sous le règne de Théodose II et attribuer la découverte à Cyrille, l'invention des reliques eut lieu sous l'empereur Arcadius et l'évêque Théophile, soit entre

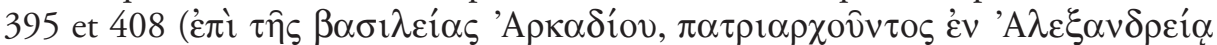

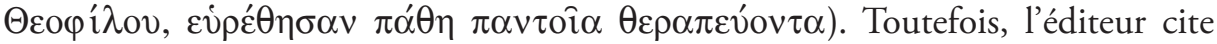
ici un manuscrit daté de 1072, offrant un récit de compromis ${ }^{122}$. Sous le règne de

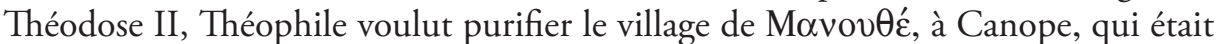

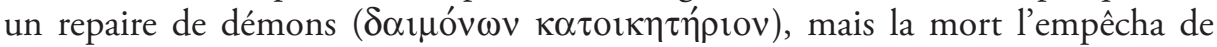
réaliser ce dessein et ce fut Cyrille qui s'en chargea:

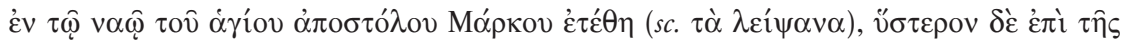

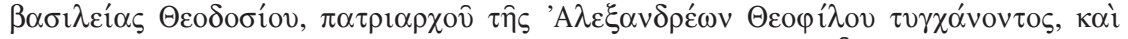

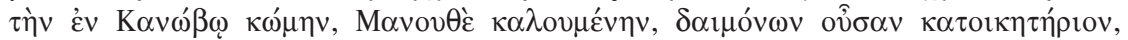

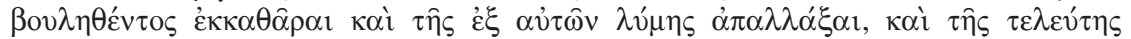

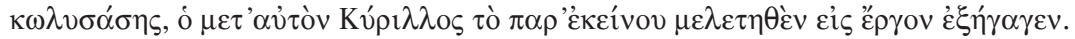

Cette version tardive de la notice suit Met., rédigée dans l'intervalle. D'après la traduction de Zylius, ce point de vue s'est transmis dans la version postmétaphrastique de $\mathrm{V} 2^{123}$.

Comme le Synaxaire est fidèle à ses sources, la mise en cause de Théophile doit refléter un état de V2 qui, sans attribuer expressément l'invention des reliques à cet

${ }^{119}$ H. Delehaye, Synaxarium Ecclesiae Constantinopolitanae (...), Bruxelles, 1902, col. 433-435 et 775-777.

${ }^{120}$ Citation de Mt. 4, 23; 9, 35, 10, 1, à propos des pouvoirs de Jésus. Elle exprime, sous la forme ramassée d'un slogan, les pouvoirs reconnus aux saints, qui sont des «imitateurs du Christ». À ce titre, nous devons la considérer comme une citation textuelle de la version de V2 connue du Synaxaire.

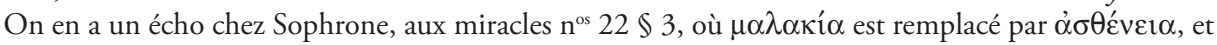
$42 \$ 4$, qui a cette fois $\dot{\alpha} \kappa \eta \delta$ í́ (léger indice d'une connaissance de cet état de V2 par Sophrone).

${ }^{121}$ La tradition faisant de Jean un médecin à l'égal de Cyr ne trouve pas d'autre appui dans les sources. Elle devait être connue à Ménouthis, puisque Sophrone, au miracle $n^{\circ} 28 \$ 11$, évoque la sonde chirurgicale des saints que l'on voyait à côté de leur cercueil.

${ }^{122}$ Synaxaire, col. 776-778 (il s'agit, sous le sigle N, d'un codex de la BNF conservant un synaxaire de mars à août); $P G 114$, col. 1249.

${ }^{123}$ Acta Sanctorum, 31 janvier, p. 1088. 
évêque, plaçait du moins la mise en place du culte en un autre temps et sous un autre patronage que ceux de Cyrille. À en juger d'après la notice prémétaphrastique du 28 juin, la version "théophilienne" s'arrêtait à la découverte des corps. Selon elle, leur vertu miraculeuse se manifesta immédiatement, ce qui signifie que le lieu d'invention se confondait avec celui du culte funéraire ultérieur. Mais ce lieu n'est pas nommé, soit qu'il ait manqué dans les exemplaires de V2 disponibles à Constantinople (hypothèse peu probable), soit que le rédacteur des notices, qui n'avait là-dessus que des informations contradictoires, n'ait pas voulu prendre parti. En tout cas, le Synaxaire ne sert pas expressément les intérêts de Ménouthis. Il se peut que la commémoraison du 28 juin ait été à l'origine célébrée dans ce sanctuaire inconnu, ce qui expliquerait que le Panégyrique ne l'ait pas retenue.

Quoi qu'il en soit du lieu, les deux légendes, théophilienne et cyrillienne, circulaient à Constantinople au $\mathrm{X}^{\mathrm{e}} / \mathrm{XI}^{\mathrm{e}}$ s. Sensible à cette contradiction, Met. a voulu les concilier. C'est ce que tente déjà Sophrone, quand il introduit ou laisse subsister dans le Panégyrique des remarques élogieuses sur Théophile, ou encore lui attribue, avec l'église des Évangélistes, une sorte de rôle préparatoire. V2, dans son état présent, suit la même ligne et va même plus loin que Sophrone en faisant de Théophile le commanditaire, non seulement de l'église des Évangélistes, mais encore de celle des Saints-Apôtres de Canope. Cela éclaire la digression de V2 sur les monuments païens de Canope: avec ses constructions, Théophile, anticipant sur l'œuvre de Cyrille, a rabaissé et éliminé un puissant paganisme local. Inversement, $\mathrm{V} 1$, toute à son orthodoxie cyrillienne, a éliminé la légende rivale, et Théophile n'y apparaît jamais. Voilà qui est fâcheux pour les tenants de l'authenticité cyrillienne des Oratiunculae, car les deux traditions ne peuvent pas être vraies en même temps, et je ne vois aucune raison de préférer l'une à l'autre. Les efforts de l'hagiographie pour les combiner sont plus édifiants que rigoureux.

\subsection{Les églises des Saints-Apôtres et des Évangélistes}

J'ajoute que les deux établissements religieux dont on vient de parler n'existaient pas sous Théophile ou Cyrille. L'un d'entre eux n'a peut-être jamais existé.

On discutera d'abord leurs formules onomastiques. Techniquement, il s'agit de "vocables" de dédicace, or cette onomastique, pour des églises à synaxe (ce qui est bien le cas ici, au moins pour celle des Évangélistes), n'avait pas cours sous Théophile et Cyrille en Égypte et à Alexandrie. On serait même bien en peine d'en trouver des attestations avant le vi ${ }^{\mathrm{e}}$ s. ${ }^{124} \mathrm{~A}$ la fin du v ${ }^{\mathrm{e}}$ s., selon Zacharie, l'église de Ménouthis n'a pas de vocable.

124 Voir J. Gascou, "Encore une fois sur l'onomastique ecclésiale ancienne ", Anthropos Laïkos, Mélanges (...) Alexandre Faivre, Fribourg, 2000, p. 119-130. 


\subsubsection{Les Saints-Apôtres}

Quant à l'établissement des Saints-Apôtres de Canope, rien de plus instructif qu'une description du site datée de 451. Elle est tirée d'une plainte déposée auprès du concile de Chalcédoine par un certain Athanase, neveu de Cyrille, contre l'évêque Dioscore, pétition qui n'a pas peu joué dans sa déposition. Fuyant les persécutions du pontife, Athanase s'est réfugié à la Métanoia:

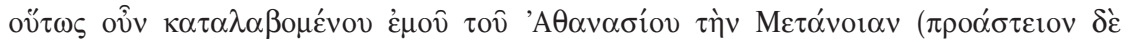

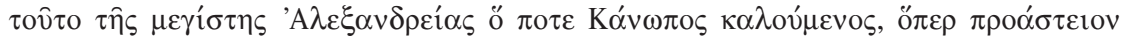

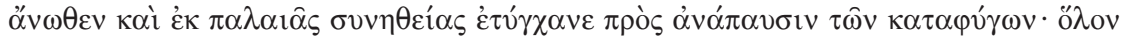

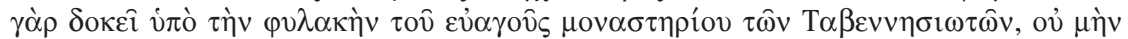

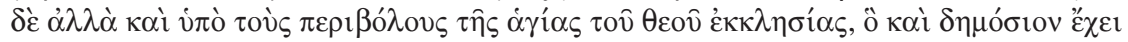

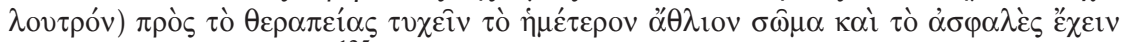

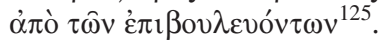

"Ainsi donc, moi, Athanase, je me rendis à la Métanoia, faubourg de l'immense Alexandrie appelé jadis Canope. Depuis longtemps, en vertu d'un antique usage, ledit faubourg était voué au soulagement des fugitifs. En effet, à ce qu'il semble, il est tout entier sous la protection du saint monastère des tabennésiotes, sans compter qu'il est aussi dans l'enceinte de la sainte église de Dieu, de même qu'il possède un bain. Je voulais que mon misérable corps y bénéficiât de soins et fût en sûreté contre mes persécuteurs ».

Athanase espérait donc trouver à la Métanoia un asile garanti par le monastère et une "sainte église de Dieu». Soit il s'agissait de l'église dont parle l'inscriptio d'Or. II, mais alors elle ne s'appelait pas église des Saints-Apôtres, soit, et peut-être plus probablement, Athanase employait le terme de "périboles " ${ }^{126}$ dans un sens figuré, voulant dire que cette asylie était garantie par l'église épiscopale d'Alexandrie (car l'expression de "sainte église de Dieu " s'appliquait de préférence aux églises épiscopales) ${ }^{127}$. Dans ce cas, l'église des Saints-Apôtres n'existait pas encore, et cela sept ans après la mort de Cyrille. Ainsi avons-nous, avec ce passage conciliaire, la preuve d'une « actualisation " d'Or. II, ou encore d'une falsification de l'inscriptio.

\subsubsection{L'église des Évangélistes}

Quant à l'église des Évangélistes, outre le problème du vocable, la forme de l'implantation du culte décrite par $O r$. II contrevient aux usages du début du $v^{\mathrm{e}}$ s. Cyrille aurait bâti le tombeau des saints à l'intérieur de l'église ('ُv $\tau \hat{n}$

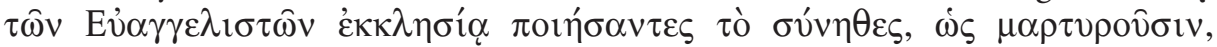
$\mu \nu \eta \mu \varepsilon \hat{i o v})^{128}$, s'exprimant même comme s'il s'agissait d'un usage habituel. Or,

\footnotetext{
${ }^{125}$ ACO II, 1, 2, p. 21.

${ }^{126}$ Ce terme est cependant employé dans le sens matériel d'enceinte d'un martyrium dans les actes du même concile ( $A C O$ II, 1, 2, p. 10).

${ }^{127}$ Dans le dernier sens vont les tentatives de l'évêque Dioscore, relatées ensuite par l'auteur, pour empêcher Athanase de bénéficier de cette asylie.

${ }^{128}$ PG 77, col. 1101.
} 
d'après l'archimandrite Chénouthé, contemporain et familier de Cyrille, cette pratique, qu'il n'approuve pas, encore qu'il l'ait observée à Constantinople et à Éphèse, n'avait pas cours en Égypte, sauf dans sa cité de Panopolis ${ }^{129}$. Notre texte reflète donc un état du culte des reliques plus tardif que celui qui prévalait à l'époque

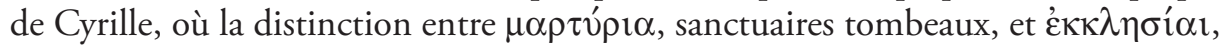
lieux de réunion, était encore bien nette. On soupçonne qu’Or. II veut justifier, en le plaçant sous la caution de Cyrille, un usage que tout le monde n'admettait pas encore, ce qui inviterait à la dater au plus tôt de la fin du ve s. ou du début du vi ${ }^{\mathrm{e}}$.

Un mystère épais environne cette église, mais une pièce d'un dossier médiéval arabe sur les huiles consacrées, que l'on n'a jamais versée au dossier, permet d'y voir plus clair. C'est en ce monument qu'à la haute époque, l'évêque d'Alexandrie, et en particulier l'illustre Théophile, c'est-à-dire son fondateur même, selon le Panégyrique et V2, procédait une fois par an, de sa main et en présence des autres évêques, au baptême des néophytes de toute l'Égypte ${ }^{130}$. Cet ancien usage est encore rapporté par une lettre d'un évêque égyptien du $\mathrm{x}^{\mathrm{e}}$ siècle tirée du même manuscrit ${ }^{131}$. Notre église y est dite cette fois "catholique" (principale; épiscopale), et possédait un baptistère ou plutôt " Jourdain », selon une terminologie prisée en Égypte.

D’origine copte et non plus chalcédonienne, le premier de ces témoignages implique une nouvelle fois Théophile, mais sans en faire un fondateur. En principe, loin d'inspirer confiance, ce nom devrait plutôt soulever la suspicion, car les Coptes ont, en d'autres occasions, abusé de la mémoire de ce pontife ${ }^{132}$. Voici pourtant une sorte de rencontre entre deux traditions religieuses ennemies. Mais cette convergence n'offre aucune garantie d'authenticité, car, sur une matière comme la liturgie du baptême, les Coptes, revenus au pouvoir après la conquête arabe, ont pu s'approprier une tradition préexistante, probablement apocryphe, mais qui ne prêtait pas à contention du point de vue des rites.

Apocryphe ou non, peu importe: l'Église d'Égypte estimait qu'à une époque reculée de son histoire, notre monument était le siège d'une cérémonie baptismale de portée nationale. En regard d'une vocation si auguste, la description de Zacharie,

${ }^{129}$ Voir L. Th. Lefort, "La chasse aux reliques des martyrs en Égypte au IV siècle ", Nouvelle Clio 6, 1954, p. 225-230, sp. p. 228. L'écrit de Chénouthé, dont Lefort donne une nouvelle traduction, est tiré de É. Amélineau, Euvres de Schenoudi, I, Paris, 1907, p. 212-220. Il est nécessairement postérieur au voyage de l'archimandrite à Éphèse, en 431, en compagnie de Cyrille. Chénouthé relève aussi qu'il n'y a aucun appui scripturaire à une telle pratique.

${ }^{130}$ L. Villecourt, "Le livre du chrême (ms. Paris arabe 100) ", Le Muséon 41, 1928, p. 57-58.

${ }^{131}$ L. Villecourt, " La lettre de Macaire, évêque de Memphis, sur la liturgie antique du chrême et du baptême, à Alexandrie ", Le Muséon 36, 1923, p. 35-36. Selon cet évêque, le baptême, à l'époque où il écrit, s'administre au monastère de Saint-Macaire au Wady al-Natrûn.

${ }^{132}$ L'archéologie lui a ôté, malgré la tradition hagiographique copte, la basilique appelée autrefois église d'Arcadius, à Saint-Mènas. Il n'a pas fondé le martyrium des Trois-Hébreux, dû à l'évêque Apollinaire (551/570) ni la chapelle des saints Côme et Damien, comme le veut Jean de Nikiou, Chronique, Zotenberg, p. 450, qui date du règne de l'empereur Justinien. 
l'anonymat, un seul prêtre, un clergé corrompu et timide, est décidément bien inadéquate.

On ne peut donc éviter une hypothèse radicale. Cette église des Évangélistes a-t-elle existé? De fait, on a du mal à croire qu'au temps de Sophrone, le culte funéraire des martyrs ait pu conduire au décri complet du nom des Évangélistes qui n'apparaît chez lui que comme un souvenir ${ }^{133}$. Il est vrai que Sophrone désigne le martyrium comme une $\dot{\varepsilon} \kappa \kappa \lambda \eta \sigma^{\prime} \alpha$ (miracles $n^{\text {os }} 14 \$ 4$ et $\left.38 \$ 11\right)$. On

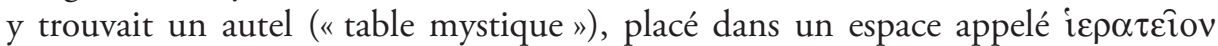
(miracle $\mathrm{n}^{\circ} 39 \$ 10$ ), mais, si l'on additionne ses détails descriptifs, on ne voit rien qui tienne aux fonctions ordinaires d'une église, par exemple la célébration de la synaxe dominicale, la prédication, les fêtes de l'année liturgique. Il est bien question de prêtres et de messe aux miracles $n^{\text {os }} 37 \$ 3-4$ et $39 \$ 10$, mais dans des contextes oniriques. Le caractère sacerdotal du vieux Geôrgios, au miracle $n^{\circ} 51$, soulève quelques difficultés. Toutefois, et dans ce cas Sophrone semble anticiper sur la tradition copte médiévale, les $\Theta \alpha v ́ \mu \alpha \tau \alpha$ évoquent à plusieurs reprises un baptistère, $\varphi \omega \tau \imath \sigma \tau \eta ́ p ı$, ou encore «Jourdain " (miracle $n^{\circ} 39 \$ 5$ ), qui était aussi le dépositoire des espèces sacramentelles. Les saints y envoyaient communier les hérétiques convertis, ce qui suffisait à leur réintégration, ou leur tenait lieu de baptême, puisque l'Église reconnaissait la validité des baptêmes hérétiques ${ }^{134}$. De même, à l'époque de Sophrone, la vie de Jean de Chypre (ou l'Aumônier) fait état d'un moine de Gaza qui, sans crainte du scandale, car il était eunuque, avait conduit une jeune juive à notre sanctuaire, où il la baptisa ${ }^{135}$. Ce Jourdain devait donc être un outil efficace et bien connu de la reconquête chalcédonienne, et de la conversion en général. Il y avait là matière à légendes. Ainsi $\mathrm{V} 1$, nous procurant un des aitia du rite baptismal local, prétend qu'à la suite de la translation des reliques, les prêtres païens de Ménouthis abjurèrent et furent immédiatement baptisés par Cyrille ${ }^{136}$.

On voit mieux à présent la portée du détail topographique, à première

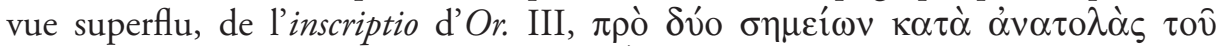

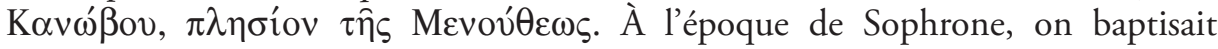
sans doute beaucoup à notre martyrium et c'était là une donnée de fait. Certains milieux chrétiens devaient pourtant s'en offusquer, y voir une concurrence déloyale au détriment des églises de la ville d'Alexandrie, peut-être même une irrégularité canonique. N'était-ce pas la meilleure façon de faire taire la critique, la meilleure légitimation que de se prétendre, pièces à l'appui, l'héritier, sur les lieux mêmes,

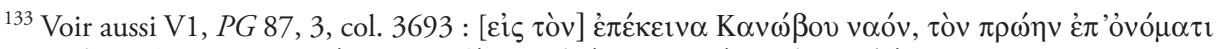

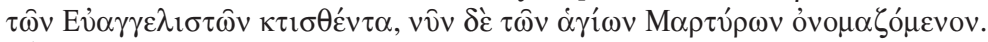

${ }^{134}$ Voir surtout les miracles $n^{\text {os }} 36 \S 14-22$ et $39 \$ 5$; allusion possible dans le miracle $n^{\circ} 37 \S 5$. Au $\mathrm{n}^{\circ} 31 \$ 6$, il ne s'agit plus d'un hérétique mais d'un blasphémateur inconscient.

${ }^{135}$ Vie de Jean de Chypre, Festugière, p. 374.

${ }^{136} P G 87,3$, col. 3696 . Le Panégyrique fait allusion au baptême des fidèles du démon (Bringel, $₫$ 29), mais en termes métaphoriques.
} 
d'une antique église pour la synaxe où l'évêque administrait le baptême à tous les Égyptiens? Le nom de Théophile, pontife fameux et incontesté, ajoutait du crédit à cette prétention.

\subsection{Ménouthis et les saints Cyr et Jean}

Selon V1, l'humanité souffrante affluait à Ménouthis depuis les " extrémités du

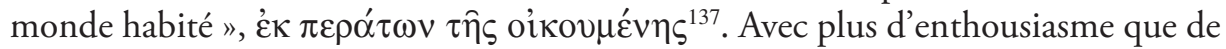
pertinence, Sophrone développe ce thème au miracle $n^{\circ} 51 \$ 1$ :

"Les prodiges accomplis par les martyrs en faveur d'étrangers, en l'occurrence de Romains, comme de Galates et de Ciliciens, d'Asiates, comme d'Insulaires et de Phéniciens, de Byzantins, comme de Bithyniens et d'Éthiopiens, de Thraces, comme de Mèdes et d'Arabes, de Palestiniens, comme de Syriens, d'Élamites et de toutes les nations qui sont sous le ciel ».

Pure propagande, exprimant plutôt les prétentions du clergé local au monopole du culte et des pérégrinations qu'une réalité, car le lien entre la dévotion à Cyr et Jean et le sanctuaire de Ménouthis n'était pas si exclusif. Bien d'autres lieux de culte, à Alexandrie, dans le reste du pays et à l'étranger revendiquaient nos saints.

\subsubsection{Un culte répandu à Alexandrie}

Dans la ville même d'Alexandrie, on compte au moins trois de ces établissements, où Cyr, saint autochtone, est plus en vue que Jean, étranger tard venu.

Le prestigieux mausolée de Saint-Marc, à l'est d'Alexandrie, près de la porte Héliaque, se voulait déjà, en tant que nécropole des évêques d'Alexandrie ${ }^{138}$, le plus gros dépositoire de dépouilles illustres de la ville. Il est à l'origine d'une hagiographie résiduelle lui attribuant les reliques de l'évêque Pierre ${ }^{\mathrm{I}}$, le plus fameux martyr de l'Église d'Égypte, dont le tombeau officiel était pourtant à l'ouest d'Alexandrie ${ }^{139}$. Son avidité s'est aussi portée sur Cyr et Jean et leurs compagnes. Un miracle arabe de Cyr, dont le cadre est Saint-Marc, montre qu'il y eut dans l'Antiquité des $\theta \alpha v ́ \mu \alpha \tau \alpha$ marciens de Cyr. Les revendications de Saint-Marc subsistaient au Moyen Âge, car le Synaxaire copte, à la commémoraison du 4 epeiph (28 juin), bien que tributaire de V1 pour les thèmes et l'expression, efface toute référence à Ménouthis et fait de Saint-Marc le lieu d'ensevelissement définitif des deux saints ${ }^{140}$. En distinguant dans l'œuvre de Cyrille, selon V1 et Sophrone, deux étapes, invention à Saint-Marc et transfert à Ménouthis, la tradition cyrillienne semble, sinon reconnaître, du

${ }^{137}$ PG 87, 3, col. 3696. Formule très voisine dans le Panégyrique (Bringel, $\$ 33$ ), encore qu'il s'agisse de la diffusion de la réputation des saints. Voir surtout le miracle $n^{\circ} 51 \$ 2$ : «Par amour pour les martyrs, nous venons les trouver depuis l'extrémité même du monde ».

${ }^{138}$ Devos, p. 172.

${ }^{139}$ Voir Gascou, Églises, p. 36-42; aux sources que j’alléguais alors, on peut joindre l'écrit syriaque nestorien dit Chronique de Séert qui va nettement dans le sens que je défendais, PO 4, p. 241-244, spéc. p. 244.

${ }^{140} P O 17$, p. 621-622; rien d'autre part sur leur tombeau à la notice du 6 mecheir ( $1^{\text {er }}$ février, jour du martyre chez les Coptes), ce qui témoigne encore d'une réticence ou d'un embarras du Synaxaire copte à reconnaître le mausolée de Ménouthis ( $P O 11$, p. 796-797). 
moins prendre en compte, dans un récit de compromis valant lot de consolation, les droits de Saint-Marc sur les reliques, droits sans doute bien défendus par son clergé, et qu'il était impossible de passer sous silence ${ }^{141}$. À ce titre, il y a lieu de penser que le lieu d'invention des reliques et de culte de la tradition théophilienne selon la version de V2 utilisée par le Synaxaire de Constantinople était Saint-Marc, et que la fête du 28 juin était à l'origine une solennité de cet établissement. Les noms des églises des Saints-Apôtres et des Évangélistes (réels ou fictifs) durent faciliter, dans la légende, l'escamotage hagiographique de Saint-Marc, car l'évangéliste Marc est considéré en Égypte comme un apôtre.

Selon V2, le martyrium des Trois-Enfants remontait à l'évêque chalcédonien Apollinaire (551/570). Situé derrière le mausolée de Jean-Baptiste, au sud

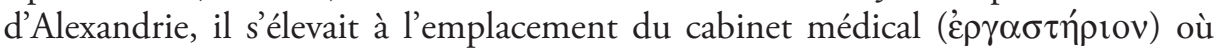
avait opéré Cyr de son vivant. De nombreuses guérisons y étaient encore dispensées et, pour souligner la vocation thérapeutique de l'endroit, on y trouvait un hôpital

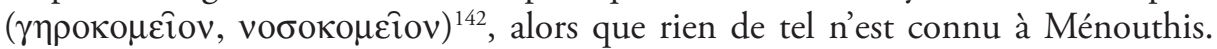
Du point de vue de la dévotion, ce n'était pas un concurrent dangereux, car V2 n'enregistre de sa part aucune revendication sur les reliques ${ }^{143}$, mais son rôle hospitalier pouvait détourner le public et accréditer l'image, inacceptable pour les partisans de la médecine thaumaturgique de Ménouthis, d’un Cyr médecin profane. Sophrone, qui n'en parle pas dans le Panégyrique, règle discrètement ses comptes avec le mausolée des Trois-Enfants au miracle n ${ }^{\circ} 28 \$ 9$ : c'était là que l'aveugle Phôteinos vendait des fruits. Pour se faire soigner, Phôteinos avait apparemment sous la main tout ce qu'il lui fallait, mais a préféré se rendre à Ménouthis. À bon entendeur!

Un miracle copte de s. Mènas évoque enfin un sanctuaire d'Apa Kyri, proche d'un milieu d'orfêvres et qui, à ce titre, devait plutôt se situer à Alexandrie même qu’à Ménouthis ${ }^{144}$.

\subsubsection{La dissémination du culte de Cyr et Jean}

Si l'on admet que toute mention de lieu, dans un écrit hagiographique, a une signification cultuelle, V2, même remaniée dans le sens souhaité par Ménouthis,

${ }^{141}$ Or. II a pourtant eu cette audace, puisque Saint-Marc n'y est mentionné qu'à propos des vierges (une fontaine, pas les corps).

${ }^{142}$ Voir J. Gascou, "Notes de papyrologie byzantine; 1) Les sanctuaires dédiés aux Trois saints Jeunes Gens ", $C d E$ 59, 1984, p. 334-335. La tradition copte attribuant l'origine de l'édifice à l'évêque Théophile n'est pas crédible (Gascou, Églises, p. 25-26). La localisation du sanctuaire " derrière " le mausolée du Baptiste est assurée par A. Mingana, "Vision of Theophilus ", Bull. of the John Rylands Library 13, 1929, p. 395.

${ }^{143}$ Il n'avait en fait aucune relique, pas même celles des Trois Hébreux, comme l'admet sans trop de difficultés son hagiographie copte. Ce n'est que par condescendance que V2 lui concède la possession d'une main d'Ananias ( $P G 87,3$, col. 3680).

${ }^{144}$ Drescher, p. $154-155$ avec la n. 2 de la p. 154. 
montre que le culte de Cyr et Jean, loin de se cantonner à Alexandrie, s'est rapidement répandu en Orient, au moins sur la côte arabique de l'Égypte, au village de Tzôté, lieu de la retraite ascétique de Cyr, à Jérusalem où Jean résidait lorsqu'il entendit parler de Cyr, et Édesse, origo de Jean. Le miracle sophronien $n^{\circ} 58 \$ 6$ relate qu'une chapelle votive leur fut élevée à Kérak. D’après le Synaxaire copte, on peut ajouter Antioche, où auraient été arrêtés les deux saints ${ }^{145}$. Un miracle d'Anastase le Perse montre que Cyr opérait dans un milieu palestinien dans les années qui ont suivi la chute de la domination sassanide ${ }^{146}$. D'après son Synaxaire,

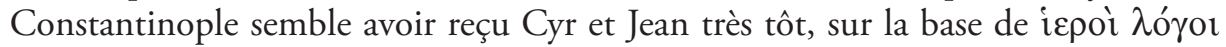
archaïques, antérieurs à V1 et Sophrone, qui se conservèrent comme des fossiles

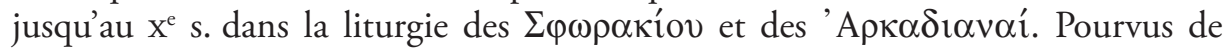
ces chapelles, les "Byzantins" n'avaient pas de raisons très fortes de se rendre en Égypte, ce qui rend d'autant plus exemplaires, dans la perspective de Sophrone, les visites à Ménouthis de deux ou trois malades de Constantinople ${ }^{147}$. D'une manière générale, il n'y a pas lieu d'exagérer la popularité du pèlerinage de Ménouthis. Ceux qui ont analysé l'origine des héros des $\Theta \alpha v ́ \mu \alpha \tau \alpha$ ont remarqué, comme c'est en général le cas pour la plupart des martyria, que le public décrit par Sophrone est surtout local ${ }^{148}$. Rien de comparable par exemple à Saint-Mènas dont la popularité internationale est un fait établi par la dispersion des trouvailles d' "eulogies ", en l'occurrence des ampoules de terre cuite ${ }^{149}$.

\subsection{Les promoteurs possibles du culte de Ménouthis}

Dans le dossier de Cyr et Jean apparaît à plusieurs reprises le faubourg alexandrin de Canope, situé à deux milles du martyrium, et donc dans son voisinage immédiat. Les données chronologiques du Panégyrique, de V1 et V2, se réfèrent, après l'empereur et le gouverneur d'Alexandrie, à un grand-prêtre païen de Canope, Kasios, Kasinos ou Kasianos, comme pour y enraciner la légende.

Canope avait, à l'époque païenne, la réputation d'une agglomération luxueuse et vouée à la fois à la religion et aux plaisirs. V2 se fait l'écho de cette tradition dans son passage sur Canope. Peu après 391, l'évêque Théophile installa sur le site une colonie de moines pachômiens, ou tabennésiotes, pour le moraliser et le

\footnotetext{
${ }^{145}$ Voir ci-dessus n. 140. Les milieux qui ont introduit Antioche dans le Synaxaire copte voulaient peut-être en même temps éliminer Canope.

${ }^{146}$ Flusin I, p. 150-153, avec la n. 108 de la p. 152 sur la large diffusion du culte de Cyr et Jean.

147 On peut citer le diacre Jôannès (miracles $n^{\text {os }} 11 \$ 5 ; 60 \$ 3$ ), le médecin Théodôros (miracle $n^{\circ}$ 60). L’argyroprate païen Agapios (miracle $n^{\circ} 32$ ) résidait déjà à Alexandrie. En revanche, un Byzantin anonyme est le héros d'un des miracles inédits du ms. Kutlumusiu 37.

${ }_{148}$ Maraval, Lieux saints, p. 106; Montserrat, p. 273-276.

149 Voir J. Witt, Menasampullen, Staatliche Museen zu Berlin - Preussischer Kulturbesitz, Skulpturensammlung und Museum für Byzantinische Kunst, Bestandskataloge, II, Werke der Alltagskultur, 1, Wiesbaden, 2000, p. 70-72.
} 
christianiser. Telle est l'origine du monastère de la Pénitence, ou Métanoia ${ }^{150}$. Ce milieu, comme nous l'avons vu en discutant l'église des Saints-Apôtres, régnait sur l'endroit. Il en a façonné à sa guise le paysage cultuel et les traditions religieuses, en plaçant par exemple sous le nom de Théophile, qui devait être en vénération particulière à la Métanoia, la fondation des Saints-Apôtres. Selon le Panégyrique, $\mathrm{V} 2$ et V1, les vierges martyres avaient Canope pour origine ${ }^{151}$. Parce qu'elle anticipe sur la vocation monastique du lieu, cette légende a de bonnes chances d'émaner de la Métanoia. L'influence des tabennésiotes éclate dans V1, qui fait de Cyr et Jean eux-mêmes des habitants de Canope ${ }^{152}$. Cyr était moine. V1 ne cherche-t-elle pas à l'enrôler à titre rétrospectif dans les matricules de la Métanoia? Un apophtegme grec et une chronique copte présentent les tabennésiotes comme des ennemis déterminés du paganisme et des temples ${ }^{153}$. De fait, selon la Vie de Sévère, ils ont participé, sur invitation de Pierre Monge, à la destruction du sanctuaire clandestin de Ménouthis. L'un d'entre eux est même le premier à pénétrer dans la salle des idoles qu'il manipule sans peur et déconsacre ${ }^{154}$. Avec cette anecdote, Zacharie leur fait en quelque sorte l'honneur d'avoir rechristianisé Ménouthis. Les Oratiunculae étaient conservées à Ménouthis, mais c’est aux tabennésiotes, dans leur version finale, qu'elles annonçaient la bonne nouvelle de l'extirpation de la Dame, de l'invention et de la translation des reliques de Cyr et Jean. Priorité flatteuse, qui tend à reconnaître à ce milieu une sorte de patronage, au moins moral, sur le martyrium. Les tabennésiotes devaient avoir un intérêt particulier à la promotion de ce culte. Du moins la vénération des reliques faisait-elle partie de leur piété155: dès leur arrivée à Canope, ils installèrent des ossements de martyrs dans les temples renversés, ce qui scandalisa le païen Eunape ${ }^{156}$. Ils avaient foi en Cyr et Jean et, selon Sophrone, allaient les visiter (miracle $\mathrm{n}^{\circ}$ 46). Peut-être même avaient-ils un droit de regard sur l'administration du mausolée, car l'économe Christodôros, qui, au temps

150 Voir en dernier lieu, sur la Métanoia, J.-L. Fournet et J. Gascou, "Moines pachômiens et batellerie ", Alexandrie médiévale 2, Études alexandrines 8, 2002, p. 23-45 (ci-après Fournet-Gascou).

151 Bringel, $\$ 18 ; P G 87,3$, col. 3681 et 3689.

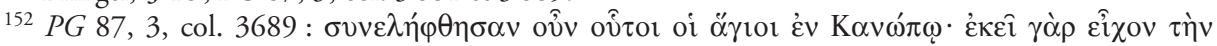

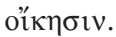

${ }^{153}$ Fournet-Gascou, p. 24, n. 3, avec renvoi à F. Nau, "Histoires des solitaires égyptiens ", ROC 13, 1908, p. 53, et T. Orlandi, Storia della chiesa di Alessandria II, Milan, 1970, p. 61.

${ }^{154}$ PO 2, p. 27-29.

${ }^{155}$ Ils aimaient aussi les objets pieux, qui sont des sortes de reliques. On trouvait ainsi dans leur église d'Alexandrie, à l'époque de la conquête arabe, un suaire de la Sainte Face, le linge dont le Christ s'était ceint lorsqu'il lava les pieds de ses disciples. Il est aussi question d'une "vénérable croix " (Jean de Nikiou, Chronique, Zotenberg, p. 515 et 574).

156 Vies des philosophes, Wright, p. 424. Delehaye, Saints d'Aboukir, p. 450, pense qu'Eunape (mort après 414) peut avoir en vue l'action de Cyrille, admettant que l'auteur ne mentionne pas expressément Ménouthis. 
de Sophrone, dirigeait l'établissement, avait exercé les mêmes fonctions auprès du martyrium alexandrin de Jean-Baptiste, qui semble avoir été lié à la Métanoia ${ }^{157}$.

De près ou de loin, l'histoire religieuse de Ménouthis implique donc la Métanoia. C'est par les tabennésiotes que se noue un accord minimal entre ces sources à première vue inconciliables, la Vie de Sévère, ouvrage monophysite ignorant Cyr et Jean, et le corpus hagiographique de Cyr et Jean, de nuance chalcédonienne.

\subsection{Quand et comment?}

Cette constatation pourrait conduire à une hypothèse historique assez voisine de celle de Duchesne. À la suite des événements relatés par Zacharie, au prix, peutêtre, de quelques contentions avec Saint-Marc, et sur le modèle de ce qui s'était passé vers 391, la Métanoia aurait mis en place le culte de Ménouthis. Elle était alors en communion avec les monophysites ${ }^{158}$. Revenue par la suite à l'orthodoxie chalcédonienne, par exemple sous le règne tracassier ou même persécuteur de Justinien, elle aurait cherché à faire oublier ses erreurs passées. Cela dut entraîner la censure de la plupart des traditions antérieures sur le martyrium, la plongée dans l'anonymat des « Pères » de V1, avec pour conséquence le vide documentaire dont se plaignait Sophrone. Cependant, une censure totale, outre ses inconvénients cultuels, aurait privé la Métanoia de ses droits moraux (et peut-être matériels) sur le sanctuaire. Placés sous le nom de Cyrille, des écrits comme les Oratiunculae présentaient le double avantage d'inscrire le martyrium dans une tradition orthodoxe, tout en le maintenant dans la mouvance du monastère de Canope.

Mais quelle confiance faut-il accorder à Zacharie? Sans doute, l'auteur se présente comme témoin oculaire de la plupart des faits, mais il ne livre pas un simple rapport, car la Vie de Sévère défend des causes. La plus affichée (et pour nous la plus lointaine) est de répondre à des attaques contre l'hérésiarque, accusé de s'être adonné aux pratiques païennes pendant sa vie d'étudiant à Alexandrie ${ }^{159}$. Zacharie veut aussi montrer que le cercle des amis et condisciples alexandrins de Sévère, dont lui-même, bien éloigné de telles complaisances, se distinguait dans l'activisme contre les païens. C'est ce que tend à établir la relation sur Ménouthis. Il s'agit en même temps de discréditer les plus éminentes figures intellectuelles de l'Alexandrie de l'époque, les philosophes païens Horapollon, Héraïskos, Ammonios, Isidore et

157 Miracle n 8 \$2. Voir mon article "Metanoia", Coptic Encyclopedy 5, p. 1608-1611, particulièrement p. 1610-1611 (on comparera le cas du tabennésiote et évêque chalcédonien Jean Talaia, qui aurait été économe de ce martyrium avant son épiscopat).

${ }^{158}$ La Métanoia est généralement considérée comme un établissement chalcédonien, mais elle a parfois changé de camp comme on le voit d'après Zacharie. De même, elle fut en communion avec l'évêque antichalcédonien Timothée III (517/535). De ses rangs sort, à n'en pas douter, le monophysite Jean le Canopite dont fait état la Vie de Sévère par Jean de Beth Aphthonia, PO 2, p. 224-225.

${ }^{159}$ PO 2, p. 7-10. On ne peut pas dire que Sévère sorte blanchi de ce récit. Il est totalement à l'écart des événements de Ménouthis. 
surtout Asclépiodote, en les associant aux "impudicités " et supercheries de l'oracle d'Isis. Ainsi mis en perspective, le récit perd de sa sincérité.

Du reste, il fourmille d'invraisemblances et thèmes convenus, empruntés soit à la littérature romanesque, soit à la polémique chrétienne traditionnelle ou encore à une sorte de "scénario " égyptien de la mise à bas des temples ${ }^{160}$. L'affaire commence par une substitution d'enfant machinée par le philosophe Asclépiodote pour faire croire qu'Isis a remédié à la stérilité de sa femme. P. Chuvin commente avec pertinence: "Une histoire d'enfant supposé (adopté en secret), banale dans l'Antiquité " ${ }^{161}$. Organisée par l'évêque Pierre sans mandat du préfet d'Alexandrie, l'expédition est un défi à l'autorité légale, concevable dans la légende, mais non pas dans la pratique administrative de l'époque. Il est vrai, selon l'auteur, que le préfet était crypto-païen ${ }^{162}$. Ce genre de diffamation n'est pas neuf dans l'histoire religieuse de l'Alexandrie tardive ${ }^{163}$. Lorsque les agresseurs se présentent à Ménouthis, ils trouvent "une maison qui était alors totalement couverte d'inscriptions païennes (hiéroglyphiques) ${ }^{164}$. Autrement dit, ce sanctuaire clandestin qui, pour fonctionner, devait acheter le silence des chrétiens du lieu ${ }^{165}$, avait néanmoins pignon sur rue, et cela à une époque de prohibition rigoureuse des cultes païens. Prévoyant le danger, les desservants de la déesse avaient obstrué l'accès à la salle cultuelle, en le maçonnant et le masquant par un meuble ${ }^{166}$. Ruse puérile. On ne comprend pas que l'étudiant Paralios, qui avait pourtant fait ses dévotions et sacrifié en ce lieu à deux reprises ${ }^{167}$, peine à en retrouver l'entrée. Après l'effraction, on retire du local, pour les convoyer à Alexandrie, vingt charges de chameau d'idoles, sans compter ce qui a été brûlé sur place ${ }^{168}$. C'est un peu beaucoup ${ }^{169}$, à moins que l'auteur ne se soit inspiré de récits alexandrins antérieurs, eux-mêmes suspects

${ }^{160}$ On trouvera, sous diverses versions, des éléments de comparaison dans le récit, probablement apocryphe, de la destruction d'un temple rural par Macaire d'Antaeopolis. Je me réfère à la traduction du copte de D. W. Johnson, A Panegyric on Macarius, Bishop of Tkôw, CSCO 416, scr. copt. 42, p. 2330 (ci-après Johnson).

${ }^{161}$ Chronique des derniers païens, Paris, 1991, p. 110 (ci-après Chuvin).

162 PO 2, p. 25.

${ }^{163}$ Sous l'évêque Cyrille, le préfet Oreste avait déjà dû se défendre de telles imputations (Socrate, Hist. eccl. 7, 14, Hansen, p. 359).

164 PO 2, p. 27.

165 Il est à l'époque banal d'expliquer la survie de l'idolâtrie par la corruption de ceux qui devraient garder les yeux ouverts, clergé et autorités: comparer Johnson, p. 23; voir le miracle nº 32 de Cyr et Jean, $\$ 1$ et 4 .

${ }^{166} P O 2$, p. 28. C'était un autel pour des sacrifices non sanglants, ce qui atténuait le crime.

${ }_{167}^{160}$ PO 2, p. 20-21.

${ }^{168}$ PO 2, p. 29 et 33. Crémation analogue dans Johnson, p. 29-30 (le prêtre local étant brûlé avec ses idoles, ce qui est encore une grave anomalie juridique).

169 De même, on trouve 306 idoles, nombre extravagant, dans le temple détruit par Macaire d'Antaeopolis (Johnson, p. 30). 
ou exagérés ${ }^{170}$. Le convoi arrive à Alexandrie avec le prêtre d'Isis. Nous sommes, comme par hasard, le dimanche de Pâques ${ }^{171}$. L'évêque Pierre a encore l'audace de " convoquer" le gouverneur et l'armée ${ }^{172}$. Il procède lui-même à un interrogatoire public du prêtre, comme s'il avait des pouvoirs de justice ${ }^{173}$. On présente les idoles au public. La plupart de ces figurations n'ont aucun rapport avec le culte d'Isis, aussi explique-t-on qu'elles provenaient d'un temple qu'avait autrefois la déesse à Memphis ${ }^{174}$. C'est en réalité un bric-à-brac littéraire où se côtoient dans l'opprobre les divinités du monde gréco-romain (outre Isis, Kronos, Dionysos, Zeus, Athéna, Artémis, Arès et Apollon) et les animaux sacrés de la tradition pharaonique (chiens, chats, singes, crocodiles, reptiles). On y trouve même le serpent qui a perdu Ève. Le public tourne les idoles en dérision, sans grande originalité ${ }^{175}$. Comme le note Chuvin: "Les gens ou l'auteur qui les fait parler, se bornent à reprendre les invectives des Pères contre l'immoralité du paganisme ${ }^{176}$.

En somme, l'authenticité de ce récit est suspecte. Je ne pense pas qu'il puisse fournir un point d'appui à l'histoire du culte de Cyr et Jean. Quand Zacharie le compose, sous l'épiscopat de Sévère (512/518), Cyr et Jean ne sont pas encore installés à Ménouthis et c'est tout ce que l'on peut en tirer.

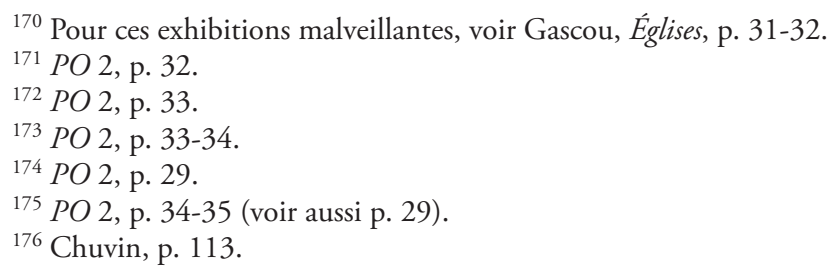

